\title{
An energy-based peridynamic model for fatigue cracking
}

\author{
Cong Tien Nguyen, Selda Oterkus*, Erkan Oterkus \\ PeriDynamics Research Centre (PDRC), Department of Naval Architecture, Ocean and \\ Marine Engineering, University of Strathclyde, Glasgow, United Kingdom
}

\begin{abstract}
Fatigue design assessment is a crucial step in the design process of ships and offshore structures. To date, the stochastic approach is commonly used to calculate the total lifetime accumulated fatigue damage and the probability of fatigue failure for the structures. Meanwhile, the details of damage initiation and propagation are infrequently investigated. In terms of predicting crack growth, the traditional approaches face conceptual and mathematical difficulties in terms of predicting crack nucleation and growth, especially for multiple crack paths because the equations in classical continuum mechanics are derived by using spatial derivatives. Peridynamics is a non-local theory using the integral equations rather than differential equations which makes it suitable for damage prediction. In this study, a novel energy-based peridynamic model for fatigue cracking is proposed. The definition of cyclic bond energy release rate range and the energy-based peridynamic fatigue equations for both phases crack initiation and crack growth phases are introduced. For validation, first, a problem of mode-I fatigue crack growth is investigated. Next, different mixed-mode fatigue damages are also investigated and the peridynamic results are compared with the experimental results.
\end{abstract}

Keywords: Peridynamics; Fatigue cracking; Crack path; Fatigue life; Cyclic bond energy release rate range 


\section{Nomenclature}

Latin Letters

$$
\begin{aligned}
& a, b, d \\
& \left(A_{1}, m_{1}\right) \\
& \left(A_{2}, m_{2}\right) \\
& \text { b } \\
& \left(B_{1(k)(j)}, n_{1}\right) \\
& \left(B_{2(k)(j)}, n_{2}\right) \\
& \bar{b}_{x(k)} \\
& \bar{b}_{y(k)} \\
& (C, M) \\
& C_{0(k)(j)} \\
& C_{1(k)(j)}
\end{aligned}
$$$$
\text { E }
$$$$
G_{c}
$$$$
g_{c}
$$$$
g_{(k)(j)}^{(N)}
$$$$
\bar{g}_{(k)(j)}
$$$$
\bar{g}_{(k)(j)}^{+}
$$$$
\bar{g}_{(k)(j)}^{-}
$$$$
g_{\infty}
$$$$
h
$$$$
H_{x}
$$$$
K_{C}
$$$$
N
$$$$
N_{c}
$$$$
N_{k}
$$$$
q
$$

Peridynamic constants for in-plane deformations.

Fatigue parameters for the phase (I) used in the PD fatigue model using cyclic bond strain range.

Fatigue parameters for the phase (II) used in the PD fatigue model using cyclic bond strain range.

The vector of body forces.

Fatigue parameters for the phase (I) used in the PD fatigue model using the cyclic bond energy release rate range.

Fatigue parameters for the phase (II) used in the PD fatigue model using the cyclic bond energy release rate range.

The external forces per unit area in $x$-direction.

The external forces per unit area in $y$-direction.

Material constants for the Paris equation using the stress intensity factor.

A coefficient that is used to calculate the energy release rate, $\bar{g}_{(k)(j)}$, for interaction between material points $k$ and $j$ in peridynamics.

A coefficient that is used to express the relationship between cyclic bond energy release rate range, $g_{(k)(j)}^{(N)}$, and the bond strain range, $\varepsilon_{(k)(j)}^{(N)}$.

Young's modulus

Critical energy release rate of material.

Average critical energy release rate for one interaction.

The cyclic bond energy release rate range at $N^{\text {th }}$ cycle of loading.

Energy release rate for interaction between material points $k$ and $j$ in peridynamics.

Energy release rate for interaction between material points $k$ and $j$ in maximum loading.

Energy release rate for interaction between material points $k$ and $j$ in minimum loading.

The cyclic bond energy release rate range corresponding to the fatigue limit $\varepsilon_{\infty}$.

Thickness of the plate.

Horizon of material point located at $\mathbf{x}$ coordinates.

Fracture toughness of material.

Number of loading cycles.

Total number of interactions passing through a unit crack area.

Total number of family members of material point $k$.

The crack length. 
R

$S_{(k)(j)}$

$S_{(k)(j)}^{+}$

$S_{(k)(j)}^{-}$

$S_{c}$

$\mathbf{t}, \mathbf{t}^{\prime}$

u

$\ddot{\mathbf{u}}$

$V_{(j)}$

$\mathbf{X}$

\section{Greek Letters}

$\delta$

$\varepsilon_{(k)(j)}^{(N)}$

$\varepsilon_{\infty}$

$\kappa$

$\lambda_{(k)(j)}^{(N)}$

$\mu$

$v$

$\xi$

$\rho$

$\phi$

$\varphi$

$\psi_{(k)(j)}$

$\omega_{(k)(j)}, \omega_{(j)(k)}$

$\vartheta_{(k)}, \vartheta_{(j)}$

The load ratio between the minimum load $P_{\min }$ and the maximum load $P_{\max }$.

The bond stretch between material points $k$ and $j$.

Bond stretch between material points $k$ and $j$ corresponding to the maximum load $P_{\max }$ of the cyclic loading.

Bond stretch between material points $k$ and $j$ corresponding to the minimum load $P_{\min }$ of the cyclic loading.

The critical bond stretch.

The peridynamic force densities.

The displacement vector.

The acceleration vector.

The volume of the material point $j$.

The vector of coordinates.

\section{Horizon size.}

The cyclic bond strain range between material points $k$ and $j$ at $N^{\text {th }}$ cycle of loading.

The fatigue limit.

Bulk modulus of the material.

The remaining life of the interaction between material points $k$ and $j$ at $N^{\text {th }}$ cycle of loading.

Shear modulus of the material.

Poisson's ratio.

Bond length in initial (undeformed) configuration.

Mass density.

Damage index to represent the local damages on the structure.

The angle between the line of interaction (between material points $k$ and $j$ in the undeformed configuration) and the $x$ axis.

Function to represent state of interaction (damaged or intact).

Micropotentials of the interaction between material point $k$ and $j$.

Dilatation of material point $k, j$, respectively.

\section{Acronyms}

CCM

FDA

FEA

PD
Classical Continuum Mechanics

Fatigue design assessment.

Finite Element Analysis.

Peridynamics/Peridynamic. 


\section{Introduction}

Ships and offshore structures are often designed with the expectation of a long lifetime in the marine environment. Due to the repetition of the loading caused by wind, wave, current, and other harsh conditions, fatigue damages can occur on the structures, which may lead to major accidents. These accidents can result in very expensive maintenance costs, crew or passenger life losses, and pollution of the marine environment. Therefore, fatigue design assessment (FDA) is one of the design drivers for ship and offshore structures.

To date, due to the randomness of the environment, the stochastic fatigue analyses are commonly used in the FDA for ship and offshore structures. In the FDA, first, the stress combination caused by hull girder loads, external wave pressure and internal cargo pressure is obtained. Subsequently, by using a suitable wave energy spectrum, the short-term stress response in irregular waves and short-term accumulated fatigue damage for the structures are predicted by using well-known Palmgren-Miner rule. Next, by using the service profile probability matrix, including wave heights, wave periods, ship headings, ship speeds, loading conditions, long-term accumulated fatigue damage, and deterministic fatigue life probability of failure are predicted. This approach is recommended by many classification societies [1-7], and it is also widely used in many practical ships and offshore structure analyses [8-12]. By using the stochastic approach, the total lifetime accumulated fatigue damage (in the long-term fatigue analysis) and the probability of failure (in the reliability fatigue damage analysis) are common outputs. Meanwhile, the details of damage initiation and propagation are still infrequently investigated.

In order to predict fatigue crack growth, the traditional finite element method (FEM) by using the remeshing techniques $[13,14]$, or various modified versions of the FEM such as extended finite element method $[15,16]$, have been used. However, one conceptual problem for classical continuum mechanics (CCM) is that it uses the partial differential equations to represent structural behaviors. Therefore, additional criteria are needed to predict crack growth speed and direction or the branching of cracks [17-20].

Peridynamics (PD) is a nonlocal theory reformulating the CCM by using integro-differential equations [21-23]. In CCM, the state of a point is only influenced by its immediate neighbors. By contrast, the behavior of a material point in PD is governed by its interactions with surrounding material points located within a finite distance $\delta$, called the horizon size. Since the integro-differential equations used in PD are valid in both continuous and discontinuous models, the theory is suitable for predicting progressive damages [24, 25].

Peridynamics can be applicable for both elastic and plastic materials and large deformation problems [26-31]. It is also applied for composite and polycrystalline materials [32-36]. The theory is also suitable for multi-physics [37, 38], coarsening and multiscale modeling [39-41]. Peridynamics is also suitable for structural idealization to analyze beam structures [42-45] or shell structures [45-49]. PD can also be combined with other well-known numerical methods such as finite element analysis (FEA) [50-53].

The first PD model for fatigue cracking is proposed by Silling and Askari [54], including three phases of fatigue failure: crack initiation (phase I), crack growth (phase II) and final failure controlled by quasi-static crack growth (phase III). Further validations for the PD model were studied by Zhang, et al. [55], Jung and Seok [56]. As proposed by Silling and Askari [54], each interaction in the PD model has its own remaining life. During the cyclic loading processes, 
the remaining life is reduced, and the reduction can be updated by using PD fatigue equations using the cyclic bond strain range.

However, in some special cases, the existing PD fatigue model [54] may face some difficulties when it is applied for elastoplastic deformation problems, beam, shell, and composite structures. In elastoplastic deformation problems, the bond strain can consist of elastic and plastic strains [29, 57]. In beam and shell structures, the bond strain consists of in-plane, shear, and bending components [42, 49]. Similarly, composite structures often exhibit coupling between in-plane and out-of-plane deformations. Therefore, deciding which strain will be used for the PD fatigue equation can be a challenge. By contrast, the energy release rate for a bond is unique and it can consist of the energy release rates for both in-plane and out-of-plane deformations [42, 49, 57-62].

Therefore, this study proposes a novel energy-based PD model for fatigue cracking. The definition of the cyclic bond energy release rate range is introduced for the first time in the PD literature. The PD fatigue equations based on the cyclic bond energy release rate range are proposed. For simplification, this study focuses on the formulations for $2 \mathrm{D}$ structures. The numerical procedures to predict fatigue crack damages are also provided and the proposed PD model is verified by considering both mode-I and mixed-mode fatigue crack growth problems.

This study is organized as follows. Section 2 presents a brief review of the PD theory for 2D structures. Section 3 presents a brief review of the existing PD model for fatigue cracking based on the cyclic bond strain range. Section 4 provides the proposed energy-based PD model for fatigue cracking by using the cyclic bond energy release rate range. The numerical procedures to predict fatigue damages are provided in Section 5. Section 6 presents the PD results for mode-I and mixed-mode fatigue damages followed by the conclusions in Section 7.

\section{Peridynamic theory for $2 D$ structures}

Peridynamics is a reformulation of the classical continuum mechanics (CCM) equations introduced by Silling [21]. As opposed to CCM, the motion of a material point is expressed in PD by using the integro-differential equation as

$$
\rho(\mathbf{x}) \ddot{\mathbf{u}}(\mathbf{x}, t)=\int_{H_{x}}\left(\mathbf{t}\left(\mathbf{u}^{\prime}-\mathbf{u}, \mathbf{x}^{\prime}-\mathbf{x}, t\right)-\mathbf{t}^{\prime}\left(\mathbf{u}-\mathbf{u}^{\prime}, \mathbf{x}-\mathbf{x}^{\prime}, t\right)\right) d V^{\prime}+\mathbf{b}(\mathbf{x}, t)
$$

The integro-differential equation given in Eq. (1) can also be represented in a discrete form as

$\rho_{(k)} \ddot{\mathbf{u}}_{(k)}=\sum_{j=1}^{N_{k}}\left(\mathbf{t}_{(k)(j)}\left(\mathbf{u}_{(j)}-\mathbf{u}_{(k)}, \mathbf{x}_{(j)}-\mathbf{x}_{(k)}, t\right)-\mathbf{t}_{(j)(k)}\left(\mathbf{u}_{(k)}-\mathbf{u}_{(j)}, \mathbf{x}_{(k)}-\mathbf{x}_{(j)}, t\right)\right) V_{(j)}+\mathbf{b}_{(k)}$

where $\rho$ represents the mass density, $\mathbf{x}$ represents the vector of coordinates, $\ddot{\mathbf{u}}$ and $\mathbf{u}$ represent acceleration and displacement vectors, respectively. In PD, the external loads are applied to the model by using the vector of body forces, $\mathbf{b}$.

As shown in Eqs. (1-2), the motion of a material point in PD is influenced by collective deformations of surrounding material points within a domain $H_{x}$, which is called horizon. In the $2 \mathrm{D}$ model, the horizon of a material point is defined as the circle with a radius $\delta$ surrounding that material point. Here, $\delta$ is called the horizon size. Material points within the horizon of a material point are called family members of that material point.

In Eq. (2), $N_{k}$ represents the number of family members of the material point $k$ and $j$ represents the family member of the material point $k$. The term $V_{(j)}$ is the volume of the 
material point $j$. The term $\mathbf{t}_{(k)(j)}$ denotes the force density that the material point $j$ exerts on the material point $k$, and $\mathbf{t}_{(j)(k)}$ corresponds to the force density that material point $k$ exerts on the material point $j$.

As studied by $[23,25,63]$ and later by $[49,64]$, the linear PD equations of motion (EOM) for a $2 \mathrm{D}$ structure can be represented as

$$
\begin{aligned}
& \rho h \ddot{u}_{(k)}=\sum_{j=1}^{N_{k}} \psi_{(k)(j)}\left[2 a d \frac{1}{\xi}\left(\vartheta_{(k)}+\vartheta_{(j)}\right)+4 b s_{(k)(j)}\right] \cos \varphi V_{(j)}+\bar{b}_{x(k)} \\
& \rho h \ddot{v}_{(k)}=\sum_{j=1}^{N_{k}} \psi_{(k)(j)}\left[2 a d \frac{1}{\xi}\left(\vartheta_{(k)}+\vartheta_{(j)}\right)+4 b s_{(k)(j)}\right] \sin \varphi V_{(j)}+\bar{b}_{y(k)}
\end{aligned}
$$

with

$$
\begin{aligned}
& \vartheta_{(k)}=d \sum_{j=1}^{N_{k}} \psi_{(k)(j)} S_{(k)(j)} V_{(j)} \\
& S_{(k)(j)}=\frac{\left(u_{(j)}-u_{(k)}\right) \cos \varphi+\left(v_{(j)}-v_{(k)}\right) \sin \varphi}{\xi} \\
& \cos \varphi=\frac{x_{(j)}-x_{(k)}}{\xi} \\
& \sin \varphi=\frac{y_{(j)}-y_{(k)}}{\xi} \\
& \xi=\sqrt{\left(x_{(j)}-x_{(k)}\right)^{2}+\left(y_{(j)}-y_{(k)}\right)^{2}}
\end{aligned}
$$

In Eqs. (3a-b), $h$ represents the thickness of the 2D structure. The terms $\bar{b}_{x(k)}, \bar{b}_{y(k)}$ represent external forces per unit area [49]. In Eq. (3c), the term $\vartheta_{(k)}$ represents the dilatation of material point $k$. In Eq. (3d), $s_{(k)(j)}$ represents the bond stretch between material points $k$ and $j$ [23, $24,49,64,65]$. The parameter $\xi$ in Eq. $(3 \mathrm{~g})$ represents the distance between material points $k$ and $j$ in the undeformed configuration. The parameter $\varphi$ represents the angle between the line of interaction (between material points $k$ and $j$ in the undeformed configuration) and the $x$ axis.

In Eq. (3a-c), the terms $a, b, d$ represent PD constants, which can be expressed as [49]

$a=\frac{E h(3 v-1)}{4\left(1-v^{2}\right)}$

$b=\frac{3 E}{(1+v) \pi \delta^{3}}$

$d=\frac{2}{\pi h \delta^{2}}$

where $E$ and $v$ represent the elastic modulus and Poisson's ratio, respectively.

For static case, the inertia terms on the left-hand sides of Eqs. (3a-b) vanishes. Therefore, Eqs. (3a-b) can be rewritten as 


$$
\begin{aligned}
& -\sum_{j=1}^{N_{k}} \psi_{(k)(j)}\left[2 a d \frac{1}{\xi}\left(\vartheta_{(k)}+\vartheta_{(j)}\right)+4 b s_{(k)(j)}\right] \cos \varphi V_{(j)}=\bar{b}_{x(k)} \\
& -\sum_{j=1}^{N_{k}} \psi_{(k)(j)}\left[2 a d \frac{1}{\xi}\left(\vartheta_{(k)}+\vartheta_{(j)}\right)+4 b s_{(k)(j)}\right] \sin \varphi V_{(j)}=\bar{b}_{y(k)}
\end{aligned}
$$

In Eqs. (3a-c) and Eq. (5), the term $\psi_{(k)(j)}$ is the damage parameter that is used to represent the state of interaction, intact or broken. This parameter can be defined as [24]

$\psi_{(k)(j)}\left(\mathbf{x}_{(j)}-\mathbf{x}_{(k)}, t\right)=\left\{\begin{array}{l}1 \text { if interaction exists, } \\ 0 \text { if no interaction. }\end{array}\right.$

The state of interaction can be determined by using either critical stretch criteria $[24,25,66$, 67] or critical energy release rate criteria [29-31, 42, 49, 68]. The damage criteria based on the critical stretch can be described as [24, 25]

$S_{(k)(j)}<S_{c} \rightarrow$ interaction exists: $\psi_{(k)(j)}=1$

$s_{(k)(j)} \geq s_{c} \rightarrow$ interaction is broken: $\psi_{(k)(j)}=0$

where $S_{c}$ represents the critical stretch. For 2D structures, the critical stretch can be calculated as $[24,25]$

$$
S_{c}=\sqrt{\frac{G_{c}}{\left(\frac{6}{\pi} \mu+\frac{16}{9 \pi^{2}}(\kappa-2 \mu)\right) \delta}}
$$

where $G_{c}$ represents the critical energy release rate of material, $\mu$ and $\kappa$ represent shear and bulk modulus of the material.

The damage criteria based on the critical energy release rate can be described as

$\bar{g}_{(k)(j)}<g_{c} \rightarrow$ interaction exists: $\psi_{(k)(j)}=1$

$\bar{g}_{(k)(j)} \geq g_{c} \rightarrow$ interaction is broken: $\psi_{(k)(j)}=0$

where $\bar{g}_{(k)(j)}$ represents the energy release rate for interaction between material points $k$ and $j$. The term $g_{c}$ represents the average critical energy release rate for one interaction.

The energy release rate for interaction between material points $k$ and $j$ can be calculated as $[29,30]$

$$
\bar{g}_{(k)(j)}=\frac{1}{2}\left(g_{(k)(j)}+g_{(j)(k)}\right)
$$

with

$$
\begin{aligned}
& g_{(k)(j)}=\frac{1}{(\Delta x) h} \omega_{(k)(j)} V_{(k)} V_{(j)} \\
& g_{(j)(k)}=\frac{1}{(\Delta x) h} \omega_{(j)(k)} V_{(j)} V_{(k)}
\end{aligned}
$$

where $\Delta x$ represents the mesh size in the uniformly discretized PD model. The terms $\omega_{(k)(j)}$ and $\omega_{(j)(k)}$ represent micropotentials of the interaction between material point $k$ and $j$. The micropotential $\omega_{(k)(j)}$ can be calculated as

$$
\omega_{(k)(j)}=\frac{1}{2 h} t_{(k)(j)} \xi s_{(k)(j)}
$$


with

$t_{(k)(j)}=\frac{2 a d}{\xi} \vartheta_{(k)}+2 b s_{(k)(j)}$

The critical energy release rate for one interaction can be computed as $[29,30]$

$g_{c}=\frac{G_{c}}{N_{c}}$

where $N_{c}$ represents the total number of interactions passing through a unit crack area, $A$. As proposed by Madenci and Oterkus [29], the interactions passing the crack tip can be counted as $1 / 2$ interaction. Meanwhile, the interaction passing a point inside the crack can be counted as 1 interaction. For a horizon size $\delta=3.015 \Delta x$, there are 48 interactions passing the unit crack surface, in which 24 interactions passing the crack tips [31, 49]. Therefore, the total number of interactions passing the unit crack surface can be counted as $N_{c}=24+24 / 2=36$.

The local damages on the structure can be represented by the damage index, $\phi$. This parameter is the ratio of broken interactions to the total number of interactions within the horizon of a material point, which can be represented as [24]

$\phi\left(\mathbf{x}_{(k)}, t\right)=1-\frac{\sum_{j=1}^{N_{k}} \psi_{(k)(j)}\left(\mathbf{x}_{(j)}-\mathbf{x}_{(k)}, t\right) V_{(j)}}{\sum_{j=1}^{N} V_{(j)}}$

\section{Peridynamics for fatigue cracking based on cyclic bond strain range}

The first PD model for fatigue cracking is developed by Silling and Askari [54]. In that model, a bond can be defined in either the crack nucleation phase (phase I) or the crack growth phase (phase II). Rapid crack growth (Phase III) can also be incorporated with the PD fatigue model when some interactions in the PD model have stretches exceeding the critical value [55]. The fatigue life of each bond is represented by its remaining life, $\lambda(\mathbf{x}, \xi, N)$ that is defined as [54]

$$
\begin{aligned}
& \lambda_{(k)(j)}^{(N=0)}=1, \quad \frac{d \lambda_{(k)(j)}^{(N)}}{d N}=-A_{1}\left(\varepsilon_{(k)(j)}^{(N)}\right)^{m_{1}} \text { with } A_{1}>0, m_{1}>0 \text { for phase (I) } \\
& \lambda_{(k)(j)}^{(N=0)}=1, \quad \frac{d \lambda_{(k)(j)}^{(N)}}{d N}=-A_{2}\left(\varepsilon_{(k)(j)}^{(N)}\right)^{m_{2}} \text { with } A_{2}>0, m_{2}>0 \text { for phase (II) }
\end{aligned}
$$

where $\left(A_{1}, m_{1}\right)$ and $\left(A_{2}, m_{2}\right)$ represent the positive fatigue parameters for the phase (I) and phase (II), respectively. The parameter $\lambda_{(k)(j)}^{(N)}$ represents the remaining life of the interaction between material points $k$ and $j$ at $N^{\text {th }}$ cycle of loading. The parameter $\varepsilon_{(k)(j)}^{(N)}$ represents the cyclic bond strain range between material points $k$ and $j$ at $N^{\text {th }}$ cycle of loading which can be defined as [54]

$$
\varepsilon_{(k)(j)}^{(N)}=\left|S_{(k)(j)}^{+}-S_{(k)(j)}^{-}\right|=\left|S_{(k)(j)}^{+}(1-R)\right|
$$

where $s_{(k)(j)}^{+}$represents bond stretch corresponding to the maximum load $P_{\max }, s_{(k)(j)}^{-}$represents bond stretch corresponding to the minimum load $P_{\min }$. The term $R$ represents the load ratio that can be defined as

$$
R=P_{\min } / P_{\max } \approx S_{(k)(j)}^{-} / S_{(k)(j)}^{+}
$$

If the fatigue limit is considered, Eq. (14a) can be rewritten for phase (I) as [54] 


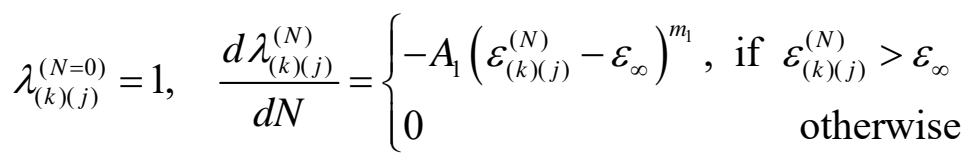

where $\varepsilon_{\infty} \geq 0$ represents the fatigue limit which is the lowest cyclic bond strain range that still results in fatigue damages. The fatigue limit, $\varepsilon_{\infty}$ can be determined from the experiment. Note that $\varepsilon_{\infty}$ can be set equal to zero if the fatigue limit is not considered [54].

By using the relations given in Eq. (17), the remaining life of a bond in phase (I) can be calculated as

$\lambda_{(k)(j)}^{(N=0)}=1, \quad \lambda_{(k)(j)}^{(N)}= \begin{cases}\lambda_{(k)(j)}^{(N-1)}-A_{1}\left(\varepsilon_{(k)(j)}^{(N)}-\varepsilon_{\infty}\right)^{m_{1}}, & \text { if } \varepsilon_{(k)(j)}^{(N)}>\varepsilon_{\infty} \\ \lambda_{(k)(j)}^{(N-1)} & \text { otherwise }\end{cases}$

By using the relations given in Eq. (14b), the remaining life of a bond in phase (II) can be calculated as

$\lambda_{(k)(j)}^{(N=0)}=1, \quad \lambda_{(k)(j)}^{(N)}=\lambda_{(k)(j)}^{(N-1)}-A_{2}\left(\varepsilon_{(k)(j)}^{(N)}\right)^{m_{2}}$

Beyond the crack growth phase (phase II), the structures can experience rapid crack growth (phase III). In this case, the traditional PD model [22-25] for damage prediction can be used. Therefore, the interaction state of a bond can be defined as

$\left\{\begin{array}{l}\lambda_{(k)(j)}^{(N)} \leq 0 \quad \text { or } \quad s_{(k)(j)} \geq s_{c} \rightarrow \psi_{(k)(j)}=0 \\ \lambda_{(k)(j)}^{(N)}>0 \text { and } \quad s_{(k)(j)}<s_{c} \rightarrow \psi_{(k)(j)}=1\end{array}\right.$

\subsection{Fatigue parameters for phase (I)}

Assuming that $\xi_{1}$ is the bond that has the largest cyclic bond strain in the structure, according to Silling and Askari [54], crack nucleation occurs when

$$
N_{1}=\frac{1}{A_{1} \varepsilon_{1}^{m_{1}}}
$$

where $\varepsilon_{1}$ represents the largest cyclic bond strain in the PD model.

If the fatigue limit, $\varepsilon_{\infty}$ is considered, Eq. (21) can be rewritten as

$$
N_{1}=\frac{1}{A_{1}\left(\varepsilon_{1}-\varepsilon_{\infty}\right)^{m_{1}}}
$$

The relations in Eqs. (21-22) can be rewritten as

$$
\log \left(\varepsilon_{1}\right)=-\frac{1}{m_{1}} \log \left(N_{1}\right)-\frac{\log \left(A_{1}\right)}{m_{1}}
$$

and

$$
\log \left(\varepsilon_{1}-\varepsilon_{\infty}\right)=-\frac{1}{m_{1}} \log \left(N_{1}\right)-\frac{\log \left(A_{1}\right)}{m_{1}}
$$

By using the relations in Eq. (23), the parameters $A$ and $m_{1}$ can be obtained from $\varepsilon-N$ test data for material as shown in Fig. 1. 


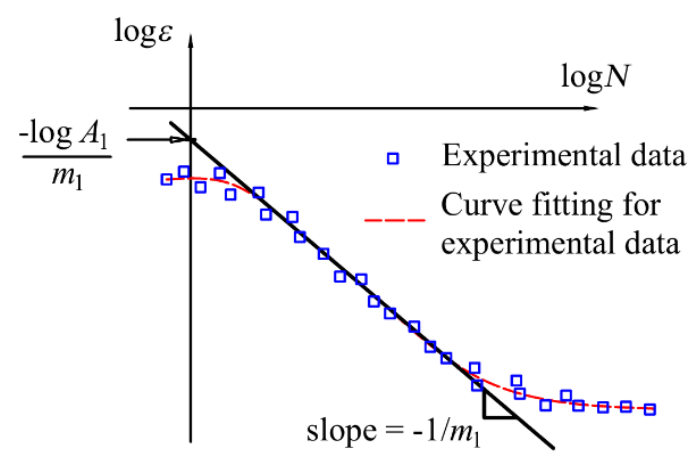

(a)

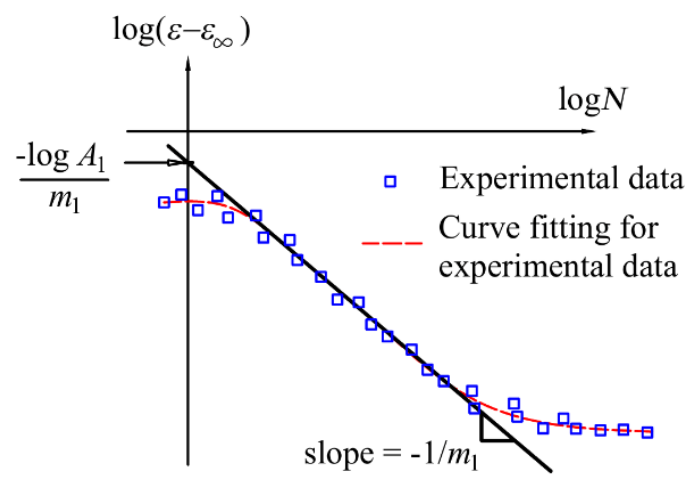

(b)

Fig. 1. Calibration phase (I) parameters $A$ and $m_{1}$ (a) without fatigue limit, (b) with fatigue limit

As shown in Fig. 1, the experimental results for $\log \varepsilon$ versus $\log N$ or $\log \left(\varepsilon-\varepsilon_{\infty}\right)$ versus $\log N$ are often represented with scatter data, shown in blue. Based on these scatter data, the fitted curves, shown in red, can be obtained. The slopes of the fitted curves are equal to $-1 / m_{1}$ . Meanwhile, the intersections of the slopes with the $\log \varepsilon$ or $\log \left(\varepsilon-\varepsilon_{\infty}\right)$ axis (the vertical axis) are equal to $-\log \left(A_{1}\right) / m_{1}$. Therefore, the fatigue parameters, $\left(A_{1}, m_{1}\right)$, for phase (I) can be obtained from the fitted curve.

\subsection{Fatigue parameters for phase (II)}

In phase (II), the fatigue crack growth follows the well-known Paris law that can be represented as [69]

$$
\frac{d q}{d N}=C \Delta K^{M}
$$

where $q$ represents the crack length and $N$ represents the number of loading cycles, $\Delta K$ represents the stress intensity factor range, $C$ and $M$ are material constants for the Paris equation using the stress intensity factor.

According to Silling and Askari [54], the parameter $m_{2}$ in Eq. (19) can be obtained directly from the material constant $M$ as

$$
m_{2}=M
$$

Meanwhile, the parameter $A_{2}$ in Eq. (19) needs to be calibrated by conducting a trial PD fatigue simulation. Details of the calibration for the parameter $A_{2}$ is presented in the appendix A which can be described as follows;

Step 1: Assume an arbitrary value for $A_{2}$ as: $A_{2}=A_{2 \text { (trial) }}$

Step 2: Conduct a PD fatigue simulation with the trial value $A_{2}=A_{2(\text { trial })}$ and calculate the crack growth rate and stress intensity (SIF) range for this trial case: $(d q / d N)_{\text {(trial) }}$ and $\Delta K_{\text {(trial) }}$.

Step 3: Plot the scatter data of $(\Delta K-d q / d N)_{\text {(trial) }}$ in the logarithmic scale and find the best-fit equation: $(d q / d N)_{\text {(trial) }}=C_{(\text {trial })} \Delta K^{M}$

Step 4: Calibrate the value for $A_{2}$ as [54] 


$$
A_{2}=A_{2 \text { (trial) }} \frac{(d q / d N)_{\text {(experiment) }}}{(d q / d N)_{\text {(trial) }}}
$$

or

$$
A_{2}=A_{2 \text { (trial) }} \frac{C_{(\text {experiment) }} \Delta K^{M}}{C_{\text {(trial) }} \Delta K^{M}}=A_{2 \text { (trial) }} \frac{C_{\text {(experiment) }}}{C_{\text {(trial) }}}
$$

where

$(d q / d N)_{\text {(experiment) }}=C_{\text {(experiment) }} \Delta K^{M}$

Here Eq. (26c) is the Paris law equation obtained from the experimental data.

Note that $m_{1}, A$ and $m_{2}$ are material constants which are independent of the horizon size. However, the parameter $A_{2}$ is dependent on the horizon size [54]. According to Silling and Askari [54], the relationship between the parameter $A_{2}$ and the horizon size $\delta$ can be presented as

$$
A_{2}(\delta)=\hat{A}_{2} \delta^{\left(m_{2}-2\right) / 2}
$$

where $\hat{A}_{2}$ is a constant and it is independent of $\delta$.

Therefore, the value of the parameter $A_{2}$ can be scaled due to the change of horizon size as follows:

Assuming that $A_{2}^{(1)}$ is the calibrated value for the parameter $A_{2}$ obtained from a trial PD simulation (see Appendix A for the calibration procedure) by using the horizon size of $\delta^{(1)}$, for example $\delta^{(1)}=3 \Delta x_{1}$ in which $\Delta x_{1}$ represents the mesh size used in the trial PD simulation.

By substituting $A_{2}^{(1)}$ and $\delta^{(1)}$ into Eq. (27), the value for the parameter $\hat{A}_{2}$ can be calculated as

$$
\hat{A}_{2}=\frac{A_{2}^{(1)}}{\left(\delta^{(1)}\right)^{\left(m_{2}-2\right) / 2}}
$$

Now, assuming that a PD fatigue simulation with a different mesh size $\Delta x_{2} \neq \Delta x_{1}$ and a horizon size of $\delta^{(2)}=3 \Delta x_{2}$ is needed. Therefore, by substituting Eq. (28) into Eq. (27) and by putting $\delta=\delta^{(2)}$, a new value of the parameter $A_{2}$ for the PD fatigue simulation with a mesh size of $\Delta x_{2} \neq \Delta x_{1}$ and a horizon size of $\delta^{(2)}=3 \Delta x_{2}$ can be obtained as

$$
A_{2}^{(2)}=\hat{A}_{2}\left(\delta^{(2)}\right)^{\left(m_{2}-2\right) / 2}=\frac{A_{2}^{(1)}}{\left(\delta^{(1)}\right)^{\left(m_{2}-2\right) / 2}}\left(\delta^{(2)}\right)^{\left(m_{2}-2\right) / 2}
$$

\section{An energy-based PD model for fatigue cracking}

In some special cases such as beam, shell, and composite structures, both in-plane and out-ofplane deformations can exist. In peridynamics, in addition to bond stretch, the definitions of other parameters including curvature and shear angle are also used to describe the behaviours of these structures $[45,49]$. Therefore, deciding which bond strain will be used for the PD fatigue equations given in Eq. (18-19) can be a challenge. By contrast, in these structures, the energy release rate for a bond is unique and it can consist of the energy release rates for both in-plane and out-of-plane deformations [42, 49, 57-62]. Therefore, in this section, a novel PD model for fatigue damage prediction based on the cyclic bond energy release rate range is 
proposed. The fatigue equation in Eq. (14) is rewritten in the energy-based form. New fatigue parameters for the proposed model are also presented.

\subsection{The cyclic bond energy release rate range}

Similar to the definition of the cyclic bond strain range proposed by Silling and Askari [54], the cyclic bond energy release rate range at $N^{\text {th }}$ cycle of loading can be defined as

$$
\boldsymbol{g}_{(k)(j)}^{(N)}=\left|\bar{g}_{(k)(j)}^{+}-\frac{R}{|R|} \bar{g}_{(k)(j)}^{-}\right|
$$

or

$$
\boldsymbol{g}_{(k)(j)}^{(N)}= \begin{cases}\left|\overline{\boldsymbol{g}}_{(k)(j)}^{+}-\overline{\boldsymbol{g}}_{(k)(j)}^{-}\right| & \text {for } R \geq 0 \\ \left|\overline{\boldsymbol{g}}_{(k)(j)}^{+}+\overline{\boldsymbol{g}}_{(k)(j)}^{-}\right| & \text {for } R<0\end{cases}
$$

where $\bar{g}_{(k)(j)}^{+}$and $\bar{g}_{(k)(j)}^{-}$represent the energy release rates for interaction between material points $k$ and $j$ in maximum and minimum loading conditions, respectively. These energy release rates can be calculated by using Eq. (10). The loading ratio $R$ is defined in Eq. (16).

Note that the bond stretches $s_{(k)(j)}^{+}$and $s_{(k)(j)}^{-}$given in Eq. (15) can be either positive or negative. However, the energy release rate $\bar{g}_{(k)(j)}^{+}$and $\bar{g}_{(k)(j)}^{-}$given in Eq. (30) are always positive. Therefore, the term $R /|R|$ is introduced in Eq. (30) to ensure the calculation of the energy release rate range can be applicable for both positive and negative load ratios.

By using the micro-potential and force density given in Eq. (11), the energy release rates for interaction between material points $k$ and $j$ given in Eq. (10) can be rewritten as

$$
\bar{g}_{(k)(j)}=\frac{1}{2}\left(\frac{2 a d}{\xi}\left(\vartheta_{(k)}+\vartheta_{(j)}\right)+4 b s_{(k)(j)}\right) \xi s_{(k)(j)} \frac{V_{(k)} V_{(j)}}{2(\Delta x) h^{2}}
$$

The energy release rate in Eq. (31) can be rewritten as

$$
\bar{g}_{(k)(j)}=\left(\frac{a d}{\xi}\left(\frac{\vartheta_{(k)}}{s_{(k)(j)}}+\frac{\vartheta_{(j)}}{s_{(k)(j)}}\right)+2 b\right) \frac{V_{(k)} V_{(j)}}{2(\Delta x) h^{2}} \xi s_{(k)(j)}^{2}
$$

or

$$
\bar{g}_{(k)(j)}=C_{0(k)(j)} S_{(k)(j)}^{2}
$$

with

$$
C_{0(k)(j)}=\left(\frac{a d}{\xi}\left(\frac{\vartheta_{(k)}}{S_{(k)(j)}}+\frac{\vartheta_{(j)}}{S_{(k)(j)}}\right)+2 b\right) \frac{V_{(k)} V_{(j)}}{2(\Delta x) h^{2}} \xi
$$

For the bond-based PD model, the term $a=0$ in Eq. (32) [25]. Therefore, Eq. (32c) can be simplified as

$$
C_{0(k)(j)}=b \frac{V_{(k)} V_{(j)}}{(\Delta x) h^{2}} \xi
$$

As can be observed from Eq. (33) for the bond-based PD model, the parameter $C_{0(k)(j)}$ is independent of loading conditions. However, for the ordinary state-based PD model, the dilatation terms exist. Therefore, as given in Eq. (32c), $C_{0(k)(j)}$ is loading dependent and it can be updated during the PD fatigue prediction. 
Note that the estimation in Eq. (32) is assumed for a bond with $s_{(k)(j)} \neq 0$. As given in Eq. (31), for a bond with $s_{(k)(j)}=0$, the energy release rate is also equal to zero, $\bar{g}_{(k)(j)}=0$. Therefore, the estimation of the energy release rate given in Eq. (32) is not needed when $s_{(k)(j)}=0$.

By using the relationship given in Eq. (32b), the cyclic bond energy release rate range in Eq. (30) can be rewritten as

$$
g_{(k)(j)}^{(N)}=\left|C_{0(k)(j)}\right|\left|\left(s_{(k)(j)}^{+}\right)^{2}-\frac{R}{|R|}\left(s_{(k)(j)}^{-}\right)^{2}\right|
$$

or

$$
g_{(k)(j)}^{(N)}=\left|C_{0(k)(j)}\right|\left|\left(1-\frac{R}{|R|} R^{2}\right)\right|\left(s_{(k)(j)}^{+}\right)^{2}
$$

By using the relation given in Eq. (15), the cyclic bond energy release rate range in Eq. (34b) can be rewritten as

$$
g_{(k)(j)}^{(N)}=\left|C_{0(k)(j)}\right|\left(1-\frac{R}{|R|} R^{2}\right) \mid\left(\frac{\varepsilon_{(k)(j)}^{(N)}}{(1-R)}\right)^{2}
$$

or

$$
g_{(k)(j)}^{(N)}=C_{1(k)(j)}\left(\varepsilon_{(k)(j)}^{(N)}\right)^{2}
$$

with

$$
C_{1(k)(j)}=\left|C_{0(k)(j)}\right|\left(1-\frac{R}{|R|} R^{2}\right) \mid \frac{1}{(1-R)^{2}}
$$

\subsection{The energy-based PD fatigue model}

Similar to the original PD fatigue model [54], in this energy-based model, the remaining life of the interaction between material points $k$ and $j$ can be represented in terms of the cyclic bond energy release rate range as

$$
\begin{aligned}
& \lambda_{(k)(j)}^{(N=0)}=1, \quad \frac{d \lambda_{(k)(j)}^{(N)}}{d N}=-B_{1(k)(j)}\left(g_{(k)(j)}^{(N)}\right)^{n_{1}} \text { with } B_{1(k)(j)}>0, n_{1}>0 \text { for phase (I) } \\
& \lambda_{(k)(j)}^{(N=0)}=1, \quad \frac{d \lambda_{(k)(j)}^{(N)}}{d N}=-B_{2(k)(j)}\left(g_{(k)(j)}^{(N)}\right)^{n_{2}} \text { with } B_{2(k)(j)}>0, n_{2}>0 \text { for phase (II) }
\end{aligned}
$$

where $\left(B_{1(k)(j)}, n_{1}\right)$ and $\left(B_{2(k)(j)}, n_{2}\right)$ represent the fatigue parameters used in the energy-based PD fatigue model for phase (I) and phase (II), respectively. The parameter $g_{(k)(j)}^{(N)}$ represents the cyclic bond energy release rate range of the interaction between material points $k$ and $j$ at the $N^{\text {th }}$ loading cycle.

If a fatigue limit is considered, the fatigue equation given in Eq. (36a) for phase (I) can be rewritten as

$$
\frac{d \lambda_{(k)(j)}^{(N)}}{d N}= \begin{cases}-B_{1(k)(j)}\left(g_{(k)(j)}^{(N)}-g_{\infty}\right)^{n_{1}}, & \text { if } g_{(k)(j)}^{(N)}>g_{\infty} \\ 0 & \text { otherwise }\end{cases}
$$


with

$g_{\infty}=C_{1(k)(j)} \varepsilon_{\infty}^{2}$

where $g_{\infty}$ represents the cyclic bond energy release rate range corresponding to the fatigue limit $\varepsilon_{\infty}$.

By integrating Eq. (37a), the remaining life of a bond in phase (I) can be calculated as

$\lambda_{(k)(j)}^{(N=0)}=1, \quad \lambda_{(k)(j)}^{(N)}= \begin{cases}\lambda_{(k)(j)}^{(N-1)}-B_{1(k)(j)}\left(g_{(k)(j)}^{(N)}-g_{\infty}\right)^{n_{1}}, & \text { if } g_{(k)(j)}^{(N)}>g_{\infty} \\ \lambda_{(k)(j)}^{(N-1)} & \text { otherwise }\end{cases}$

By integrating Eq. (36b), the remaining life of a bond in phase (II) can be calculated as

$\lambda_{(k)(j)}^{(N=0)}=1, \quad \lambda_{(k)(j)}^{(N)}=\lambda_{(k)(j)}^{(N-1)}-B_{2(k)(j)}\left(g_{(k)(j)}^{(N)}\right)^{n_{2}}$

Similar to the damage criteria given in Eq. (20), the state of interaction in the energy-based model can be defined as

$\left\{\begin{array}{lll}\lambda_{(k)(j)}^{(N)} \leq 0 & \text { or } \quad \bar{g}_{(k)(j)} \geq g_{c} \rightarrow \psi_{(k)(j)}=0 \\ \lambda_{(k)(j)}^{(N)}>0 & \text { and } \quad \bar{g}_{(k)(j)}<g_{c} \rightarrow \psi_{(k)(j)}=1\end{array}\right.$

\subsection{Fatigue parameters $\left(B_{1(k)(j)}, n_{1}\right),\left(B_{2(k)(j)}, n_{2}\right)$}

In this section, the relationships between the fatigue parameters in the proposed energy-based PD model, $\left(B_{i(k)(j)}, n_{i}\right)$ and the fatigue parameters in the cyclic bond strain model, $\left(A_{i}, m_{i}\right)$ are presented. The values of $\left(B_{i(k)(j)}, n_{i}\right)$ can be obtained indirectly from the experimental data through the calibrations for $\left(A_{i}, m_{i}\right)$. Note that, in this section, $i=1$ denotes phase (I) and $i=2$ denotes phase (II).

By comparing Eq. (36) with Eq. (14), the relationships between fatigue parameters in the energy-based model, $\left(B_{i(k)(j)}, n_{i}\right)$ and the cyclic bond strain model, $\left(A, m_{i}\right)$ can be obtained as $A_{i} \varepsilon_{(k)(j)}^{m_{i}}=B_{i(k)(j)} g_{(k)(j)}^{n_{i}}$

or

$\log \left(A_{i}\right)+m_{i} \log \left(\varepsilon_{(k)(j)}\right)=\log \left(B_{i(k)(j)}\right)+n_{i} \log \left(g_{(k)(j)}\right)$

On the other hand, the relation given in Eq. (35b) can be rewritten as

$$
\log \left(g_{(k)(j)}\right)=\log \left(C_{1(k)(j)}\right)+2 \log \left(\varepsilon_{(k)(j)}\right)
$$

Therefore, by using the relation given in Eq. (42), the relations in Eq. (41b) can be rewritten as $\log \left(A_{i}\right)+m_{i} \log \left(\varepsilon_{(k)(j)}\right)=\log \left(B_{i(k)(j)}\right)+n_{i} \log \left(C_{1(k)(j)}\right)+2 n_{i} \log \left(\varepsilon_{(k)(j)}\right)$

or

$\log \left(\frac{A_{i}}{\left(C_{1(k)(j)}\right)^{n_{i}}}\right)+m_{i} \log \left(\varepsilon_{(k)(j)}\right)=\log \left(B_{i(k)(j)}\right)+2 n_{i} \log \left(\varepsilon_{(k)(j)}\right)$

In order to ensure Eq. (43b) is correct for every interaction, the following relations between fatigue parameters in the energy-based PD model, $\left(B_{i(k)(j)}, n_{i}\right)$ and the cyclic bond strain PD model, $\left(A, m_{i}\right)$ can be obtained as

$n_{i}=m_{i} / 2$ 
$B_{i(k)(j)}=\frac{A_{i}}{\left(C_{1(k)(j)}\right)^{m_{i} / 2}}$

Therefore;

$n_{1}=m_{1} / 2$

$B_{1(k)(j)}=\frac{A_{1}}{\left(C_{1(k)(j)}\right)^{m_{1} / 2}}$

and

$n_{2}=m_{2} / 2$

$B_{2(k)(j)}=\frac{A_{2}}{\left(C_{1(k)(j)}\right)^{m_{2} / 2}}$

\subsection{Phase transition}

According to Silling and Askari [54], the PD bond strains in the nucleation phase can agree with the measured strain data. However, in the growth phase, the actual process zone at a crack tip is usually smaller than the PD continuum-level model. Therefore, bond strains in phase (II) could be fictitious. As a result, the transition from phase (I) to phase (II) might not be smooth because the bond strain can suddenly become fictitious.

In order to avoid the above phenomenon for phase transition, Silling and Askari [54] proposed a method based on the information of the damage index $\phi$ at each material point and its family members. Specifically, phase (I) fatigue equation given in Eq. (18) or Eq. (38) for a given material point $k$ is valid, if all material points within its horizon (including itself) has the damage index $\phi<\phi_{0}$. By contrast, the phase (II) fatigue equation given in Eq. (19) or Eq. (39) is valid when material point $k$ or at least one of its family members have $\phi \geq \phi_{0}$. According to Silling and Askari [54], $\phi_{0}$ can be chosen as $\phi_{0}=0.5$. In this study, the same approach is used. However, as shown in Fig. 2 for an example of a plate with a crack, material points located along the crack have the damage index of $\phi=0.398$ for the horizon size of $\delta=3 \Delta x$. It means that the plate can be completely split when the damage indexes of material points reach the value of $\phi \geq 0.398$. Therefore, in this study, instead of using $\phi_{0}=0.5$ as suggested by Silling and Askari [54], the value $\phi_{0}=0.398$ is used to decide the phase transition for each bond in two-dimensional models.

If some interactions have energy release rates exceeding the critical value, $\bar{g}_{(k)(j)} \geq g_{c}$, the fatigue simulation is stopped and the conventional PD model for damage prediction (phase III) [22-25] can be used. 


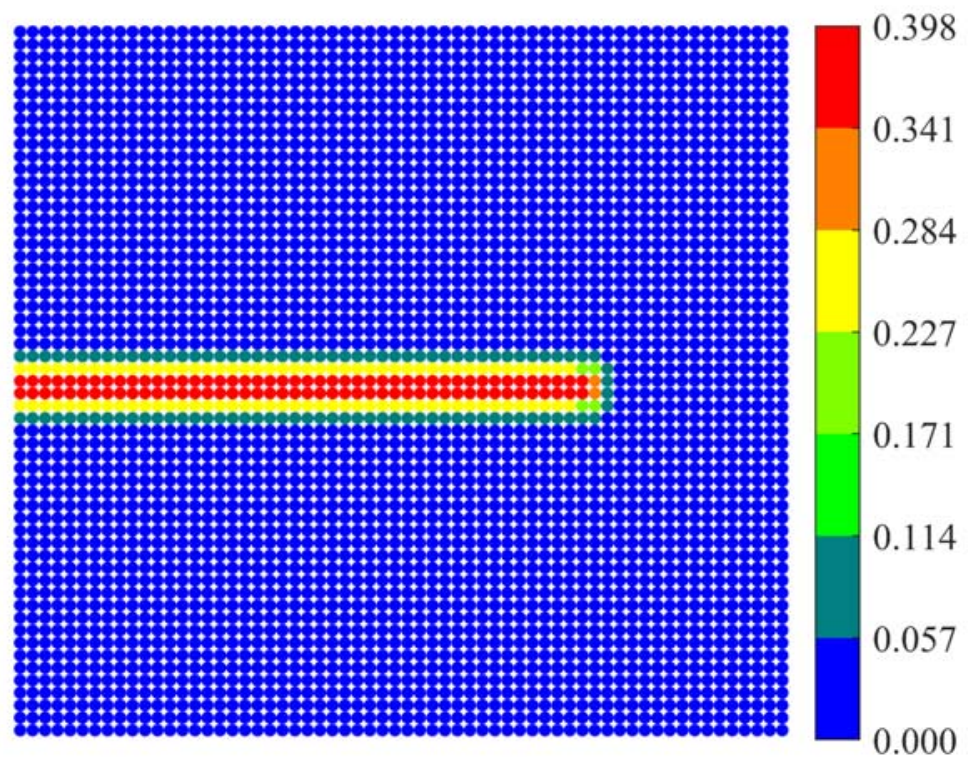

Fig. 2. Variation of damage index, $\phi$, on in plate with a pre-existing crack

\section{Numerical procedure}

In the PD model, the domain is uniformly discretized with material points associated with specific volumes. The behavior of the structure subjected to the statically extreme loading $P=P_{\max }$ is obtained by solving the PD equations of motion given in Eq. (6). Details of the solution technique for static loading conditions are presented in Appendix B.

In the cyclic bond strain range PD fatigue model [54], the remaining life of each interaction is updated by using Eq. (18) for phase (I) and Eq. (19) for phase (II). The state of interactions can be updated by using Eq. (20). In the energy-based PD fatigue model, the remaining life of each interaction is updated by using Eq. (38) for phase (I) and Eq. (39) for phase (II). The state of interactions can be updated by using Eq. (40). The numerical procedure for fatigue damage predictions by using the cyclic bond strain PD model and by using the energy-based PD model are shown in Fig. 3 and Fig. 4, respectively. 


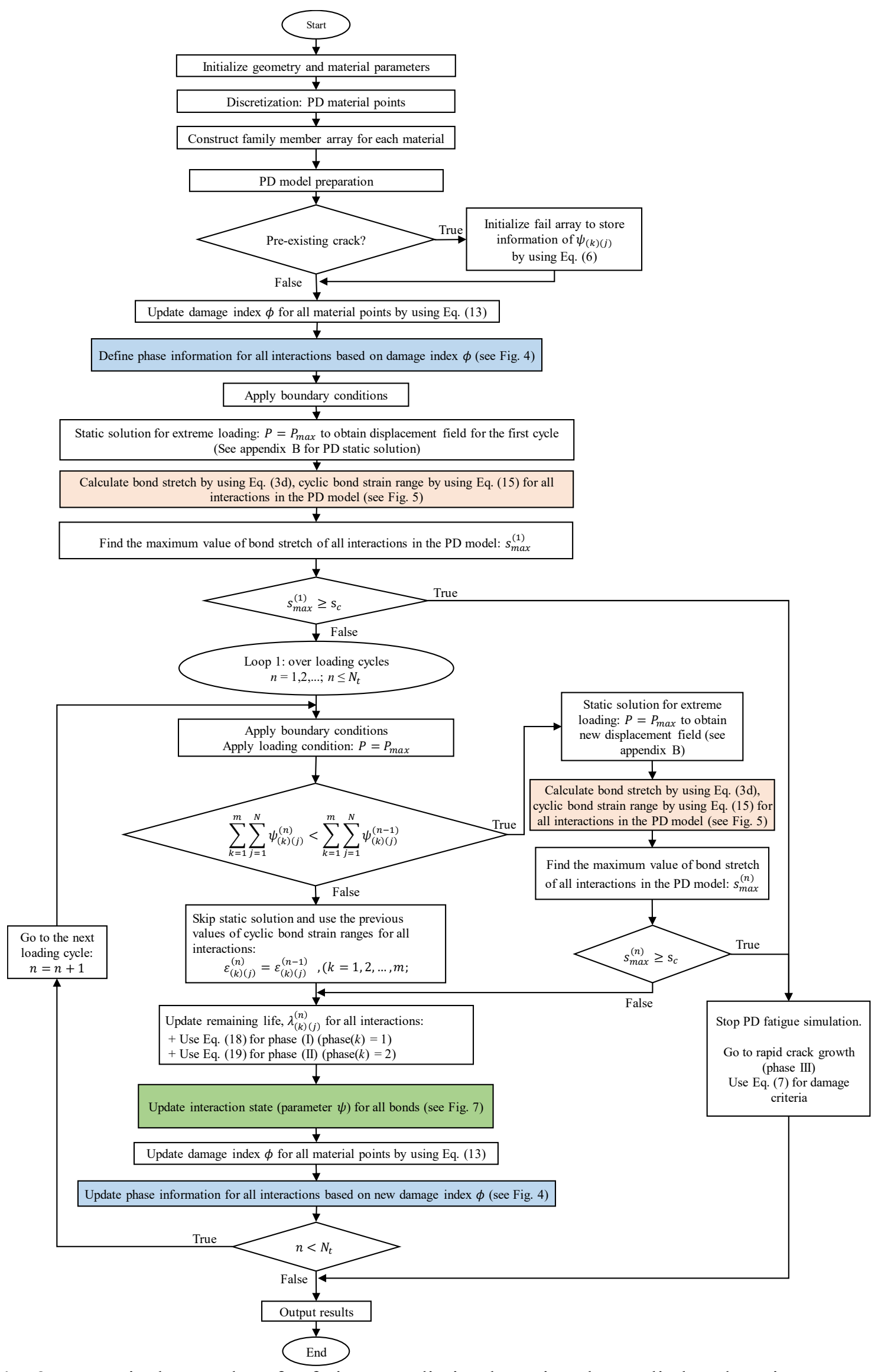

Fig. 3. Numerical procedure for fatigue prediction by using the cyclic bond strain range PD model ( $m$ represents the total number of material points) 


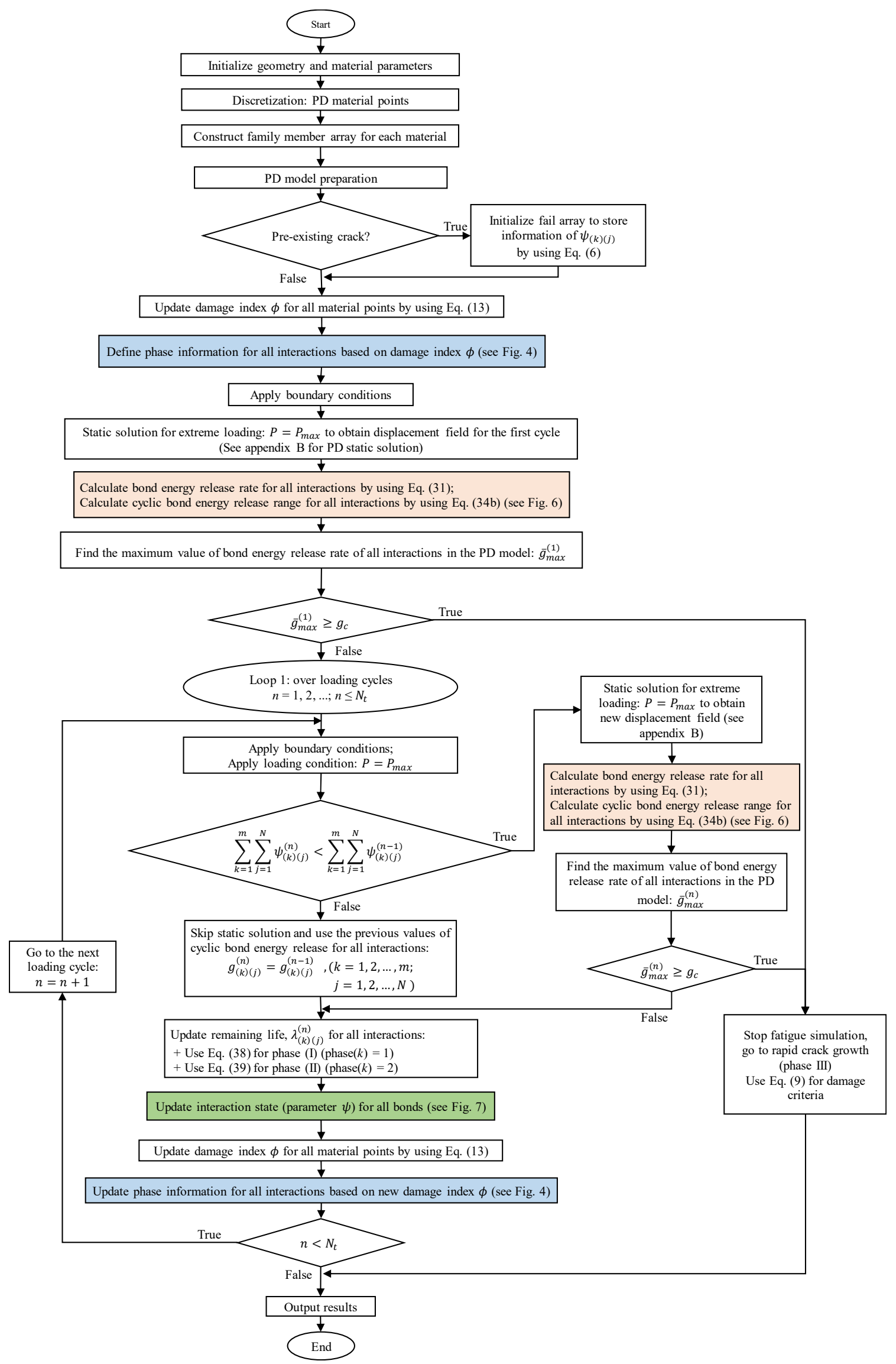

Fig. 4. Numerical procedure for fatigue prediction by using the energy-based PD model $(\mathrm{m}$ represents the total number of material points) 
As shown in Fig. 3 and Fig. 4, in order to decide whether the static solution is skipped or not, the summation of all interaction states is compared with its value in the previous load cycle.

$$
\begin{aligned}
& \Pi^{(n)}=\sum_{k=1}^{m} \sum_{j=1}^{N_{k}} \psi_{(k)(j)}^{(n)} \\
& \Pi^{(n-1)}=\sum_{k=1}^{m} \sum_{j=1}^{N_{k}} \psi_{(k)(j)}^{(n-1)}
\end{aligned}
$$

where $\psi_{(k)(j)}^{(n)}$ represents the interaction state of the bond between material points $k$ and $j$ at load cycle $n$ and $\psi_{(k)(j)}^{(n-1)}$ represents the interaction state of the bond between material points $k$ and $j$ at load cycle $n-1$.

If the summation of all interaction states at the current cycle is smaller than its value at the previous cycle, $\Pi^{(n)}<\Pi^{(n-1)}$, it means that some new bonds are broken. Therefore, the static solution for extreme loading $P=P_{\max }$ needs to be solved to obtain a new displacement field. Otherwise, there is no newly broken bond in the PD model and the static solution can be skipped. Therefore, the solution results for the previous loading cycle $n-1$ can be used for the current loading cycle $n$.

As shown in Fig. 3 and Fig. 4, the phase information for each interaction is updated after each loading cycle to decide whether phase (I) or phase (II) fatigue equation is valid for each interaction. The procedure is presented in Fig. 5. Similarly, the interaction states for all bonds in the PD model are also updated after each loading cycle as shown in Fig. 3 and Fig. 4. Details are presented in Fig. 8.

As shown in Fig. 3 for the fatigue prediction by using the cyclic bond strain range model [54], stretches and strain ranges of all interactions in the PD model are calculated at each loading cycle. Details of calculating bond stretches and cyclic bond strain ranges are presented in Fig. 6.

Fig. 4 presents the fatigue prediction procedure by using the energy-based PD model, the energy release rate and the cyclic bond energy release rate range are calculated for each interaction at each loading cycle as presented in Fig. 7. 


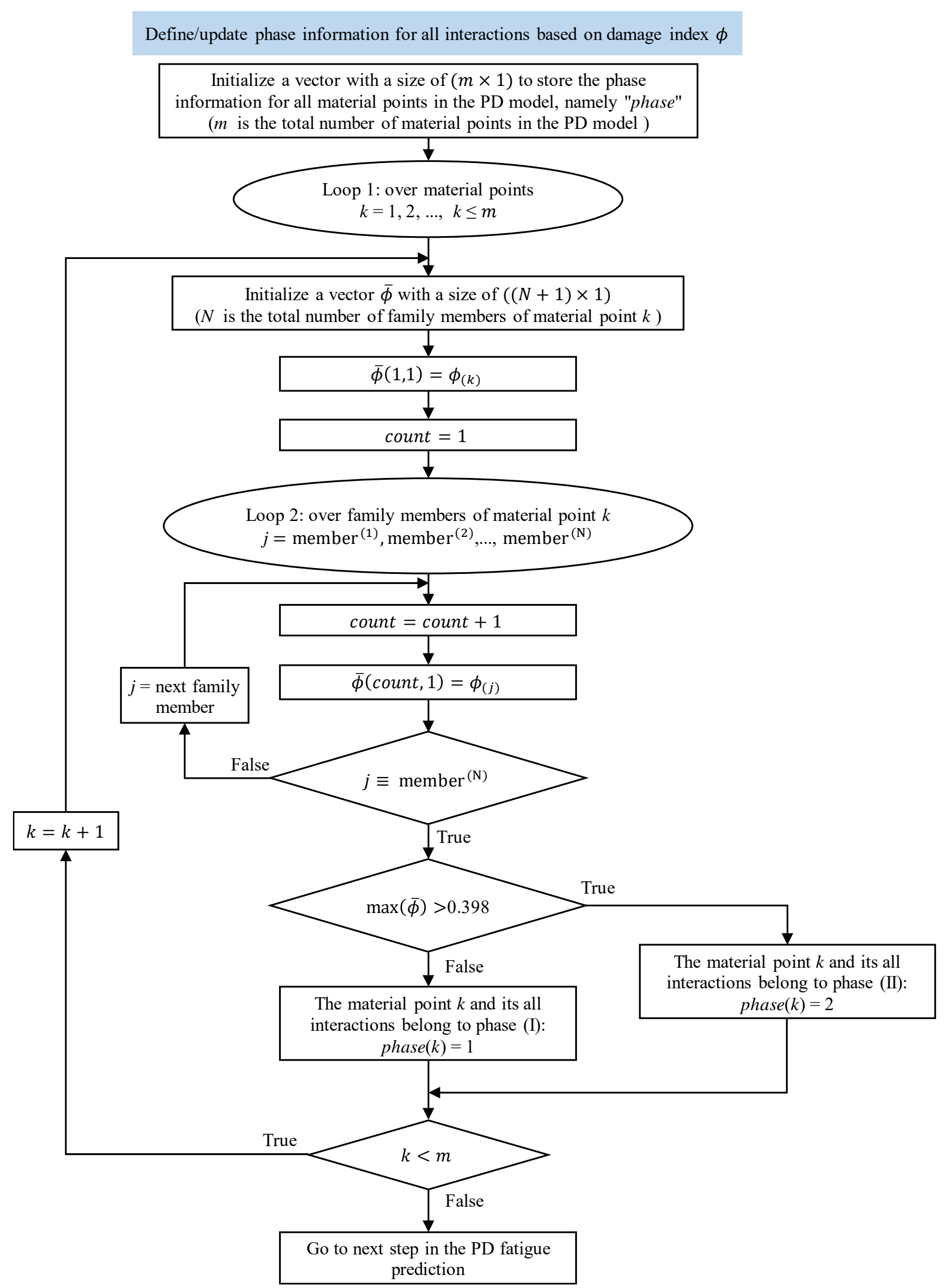

Fig. 5. Define/update phase information for all interactions in the PD model 


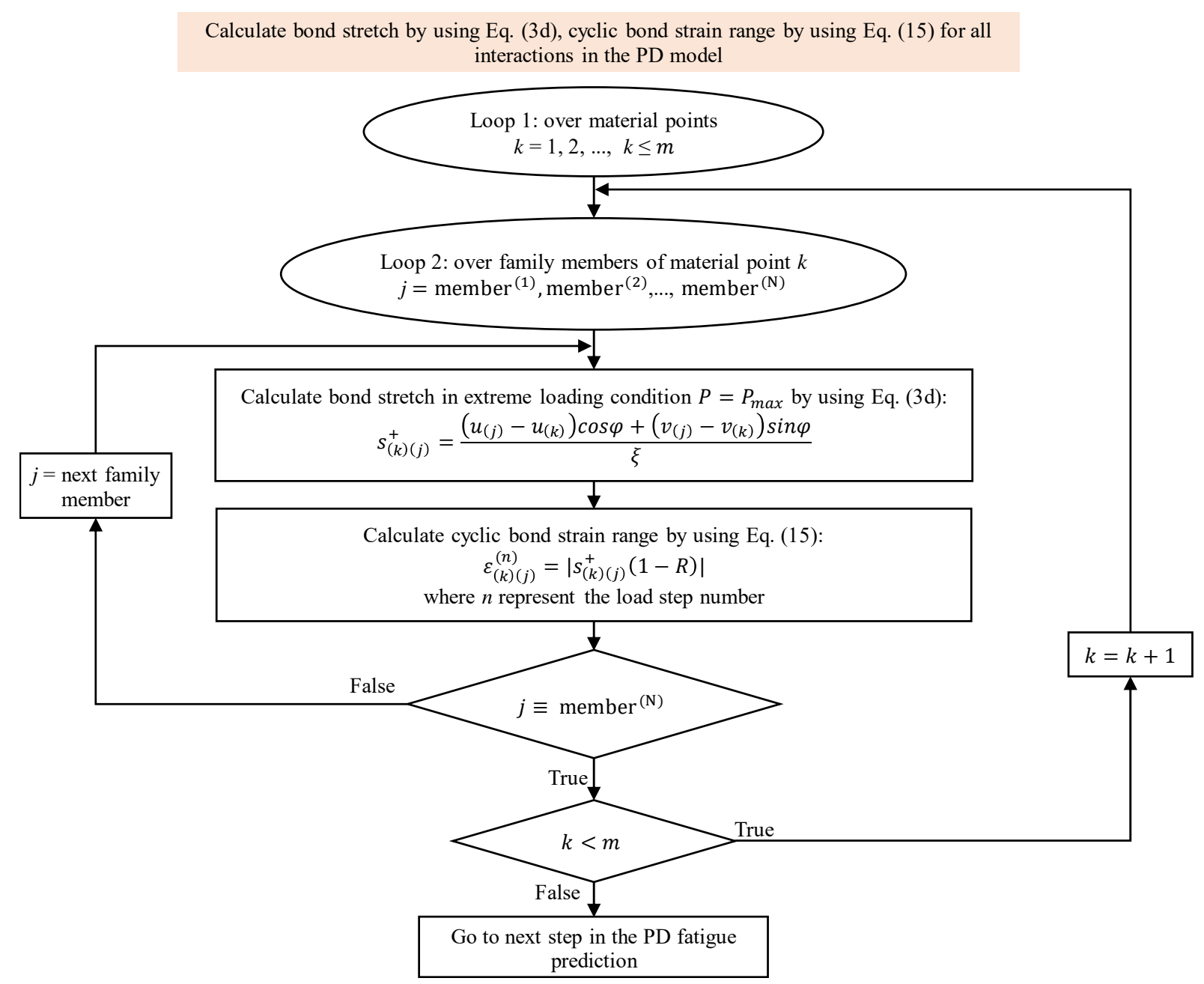

Fig. 6. Calculate bond stretches and cyclic bond strain ranges for all interactions in the PD model 


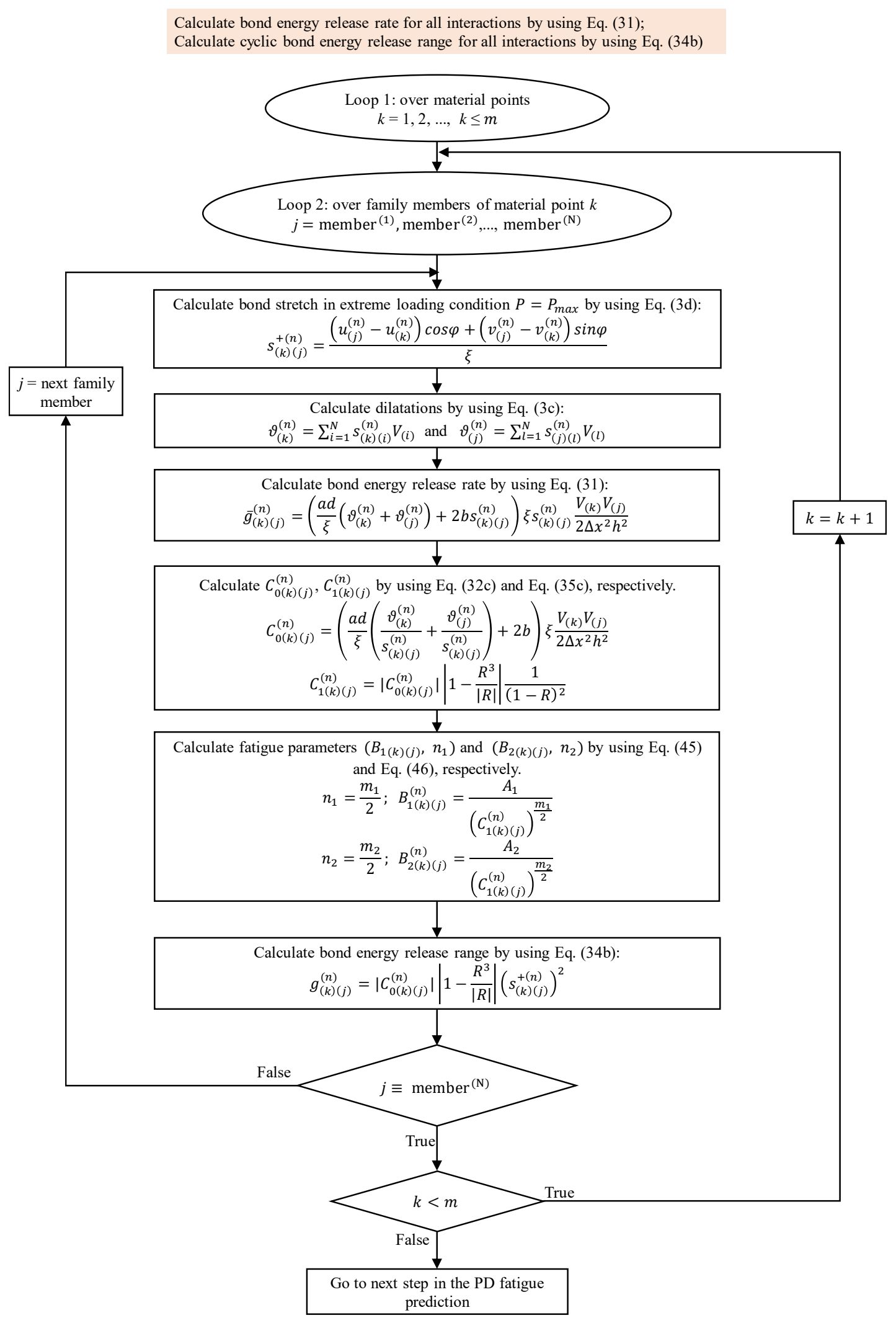

Fig. 7. Calculate bond energy release rate, cyclic bond energy release rate range (The fatigue parameters $\left(B_{1(k)(j)}, n_{1}\right),\left(B_{2(k)(j)}, n_{2}\right)$ are calculated to update the remaining life on the next step in the PD fatigue simulation in Fig. 4) 


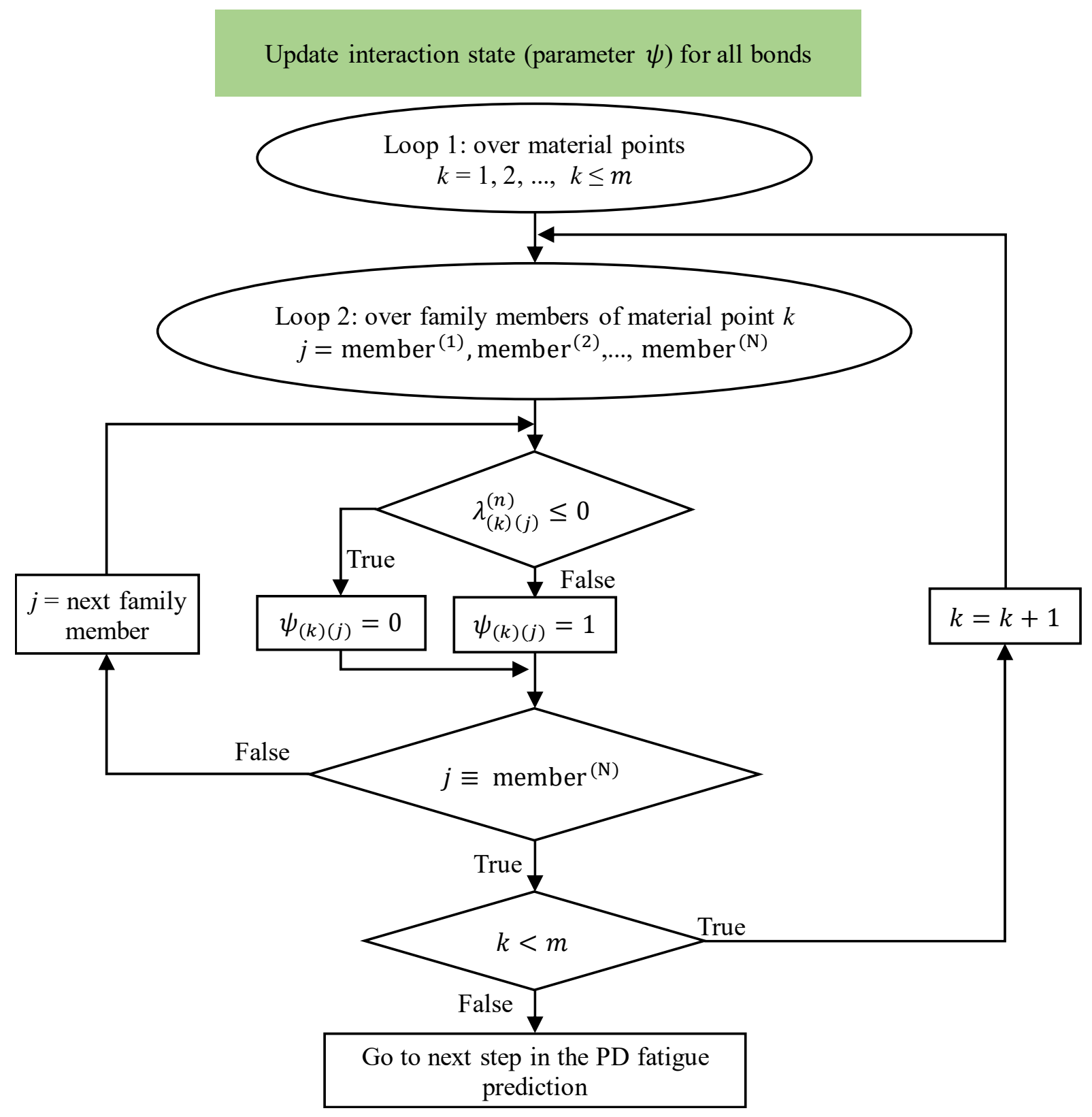

Fig. 8. Update interaction states for all bonds

\section{Numerical results}

In this section, mode-I and mixed-mode fatigue damages on $2 \mathrm{D}$ structures are predicted by using the proposed energy-based PD fatigue model as shown in Fig. 10 and Fig. 13. The structures are made of aluminum 6061-T6 with the elastic modulus of $E=68 \times 10^{9} \mathrm{~N} / \mathrm{m}^{2}$, Poisson's ratio of $v=0.33$ [70], and fracture toughness of $K_{C}=48.7 \times 10^{6} \mathrm{MPa} \sqrt{\mathrm{m}}$ [71].

The fatigue parameters for phase (I) can be calibrated by using the experimental results $(\varepsilon-N$ curve) provided in [72]. In this study, the $\varepsilon-N$ data provided in [72] is reconstructed and plotted on a log-log scale as shown in Fig. 9. Based on the calibration shown in Fig. 9, the fatigue parameters for phase (I) (without the consideration of fatigue limit) are obtained as $m_{1}=2.29153$ 
$-\frac{\log A_{1}}{m_{1}}=-0.87975$

or

$A_{1}=103.7465$

Therefore, the fatigue parameters for phase (I) in the energy-based PD model can be obtained by using Eq. (45) as

$$
\begin{aligned}
& n_{1}=\frac{m_{1}}{2}=1.145765 \\
& B_{1(k)(j)}=\frac{A_{1}}{\left(C_{1(k)(j)}\right)^{m_{1} / 2}}
\end{aligned}
$$

where $C_{1(k)(j)}$ is obtained by using Eq. (35c).

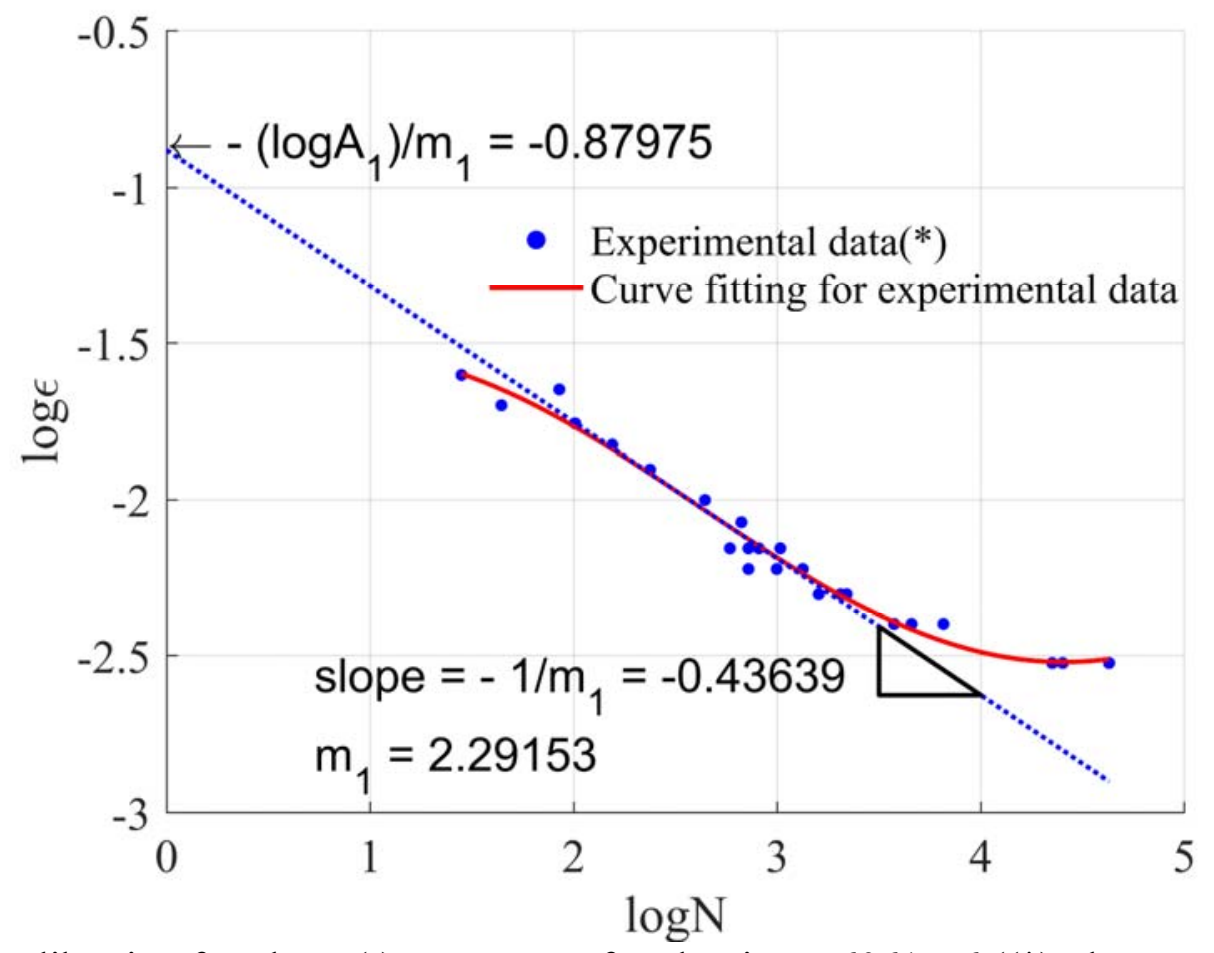

Fig. 9. Calibration for phase (I) parameters for aluminum 6061-T6 ((*): the experimental data is reproduced from [72])

\subsection{Mode I fatigue crack propagation}

In this section, the mode-I fatigue problem is investigated as shown in Fig. 10(a). The dimensions of the plate are shown in Fig. 10(a) and the PD discretized model is shown in Fig. 10 (b). The plate is subjected to cyclic loading with maximum loading $P_{\max }=14 \mathrm{kN}$, load ratio $R=0.1$, and loading frequency of $10 \mathrm{~Hz}[70]$.

In PD, the model is uniformly discretized with mesh size $\Delta x=6 \times 10^{-4} \mathrm{~mm}$ and the horizon size $\delta=3.015 \Delta x$ is used. Since the problem is symmetric, the fixed boundary conditions at two material points located at $(1.25 W, \Delta x / 2)$ and $(1.25 W,-\Delta x / 2)$, shown in black in Fig. 10(b), are assumed. 
In order to apply loading conditions, first, material points located inside the cut-outs, shown in red in Fig. 10(b), are assumed as rigid with the elastic modulus of $E_{\text {rigid }}=200 E$. Next, the extreme load $P=14 \times 10^{3} \mathrm{~N}$ is applied to the material points located at the centers of the cutouts as shown in Fig. 10(b).

The fatigue parameter $m_{2}=2.6183$ is obtained from the experimental results given by Sajith, et al. [70]. Therefore, the phase (II) fatigue parameter for the energy-based PD model $n_{2}=1.3092$ is used.

In order to obtain values for parameters $A_{2}$ and $B_{2(k)(j)}$, a trial value $A_{2(\text { trial })}$ needs to be assumed and the corresponding value $B_{2(k)(j)}^{(\text {trial })}$ is obtained for each interaction by using Eq. (46b). A trial fatigue simulation using $\left(B_{1(k)(j)}, n_{1}=1.145765\right)$ obtained from Eq. (49) for phase (I) and $\left(B_{2(k)(j)}^{\text {(trial) }}, n_{2}=1.3092\right)$ for phase (II) is conducted to calculate the fatigue crack growth rate $(d q / d N)_{(\text {trial })}$ and the SIF range $\Delta K_{\text {(trial) }}$ (see appendix A). The best-fit equation with the form of $(d q / d N)_{\text {(trial) }}=C_{(\text {trial) }} \Delta K^{M}$ is obtained by using $(d q / d N)_{\text {(trial) }}$ and $\Delta K_{\text {(trial) }}$ values. Next, by comparing $(d q / d N)_{(\text {trial) }}$ with the experimental values $(d q / d N)_{\text {(experiment) }}$, the calibrated value of the parameter for $A_{2}$ is obtained by using Eq. (26b) and the parameter $B_{2(k)(j)}$ is obtained for each interaction by using Eq. (46b).

In this example, the values of $A_{2}$ and $B_{2(k)(j)}$ are obtained after two steps of calibration. In the first calibration step, to save the computational cost for the trial simulation, a large trial value of $A_{2(\text { trial 1) }}=10^{4}$ is assumed. Next, by using the calibration method discussed above and in appendix A, the first calibrated value of $A_{2}^{(\text {calibrated 1) }}=1174$ is obtained. The value of $B_{2(k)(j)}^{(\text {calibrated } 1)}$ is also obtained by using Eq. (46b). However, the results obtained from the PD fatigue simulation using $\left(B_{1(k)(j)}, n_{1}=1.145765\right)$ and $\left(B_{2(k)(j)}^{\text {(calibrated 1) }}, n_{2}=1.3092\right)$ still have significant differences with the experimental results in terms of the $q-N$ curve. Therefore, the second calibration step with $A_{2(\text { trial 2) }}=1174$ is conducted, and the final calibrated value of $A_{2}=1055$ is obtained. Therefore, the fatigue parameter $B_{2(k)(j)}^{(\text {calibrated } 2)}$ is finally obtained by using Eq. (46b). 


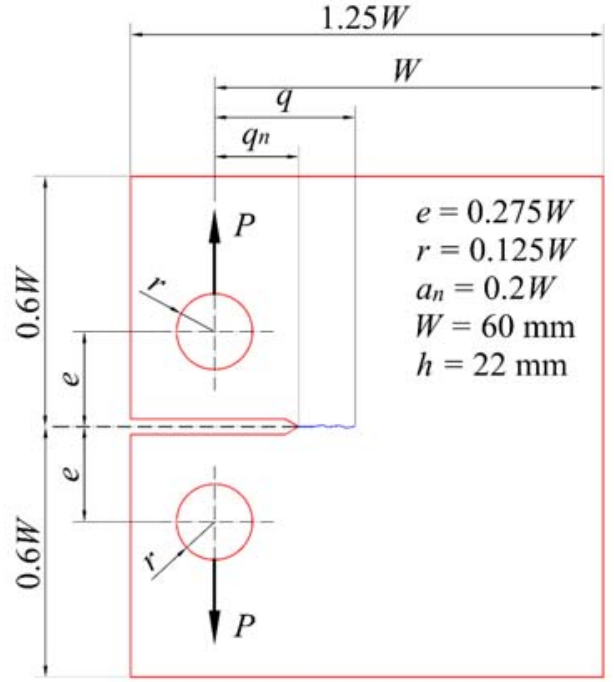

(a)

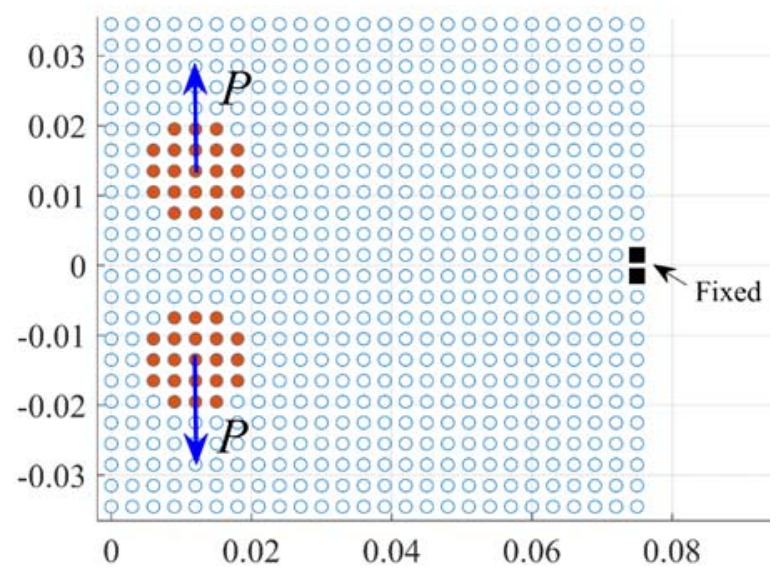

(b)

Fig. 10. Mode I fatigue problem (a) geometry (b) PD discretized model

Fig. 11 shows the fatigue damage evolution on the plate under mode-I loading condition. After 2000 loading cycles, the crack starts propagating as shown in Fig. 11(a). As expected, the crack propagates along its initial direction toward the right edge of the plate as shown in Fig. 11(bd). After 40850 loading cycles, the crack reaches the location at $x=0.0522 \mathrm{~m}$ as shown in Fig. 11(d). Fig. 12 shows crack length, $q$ versus load cycle, $N$ for fatigue crack growth of the plate. As can be observed from the figure, the PD prediction results have good agreement with the experimental results [70]. The crack length, $q$ versus load cycle, $N$ predicted by the PD model agrees very well with the experimental curve for the first 30000 cycles. Later, the predicted crack growth is slightly quicker than the experimental results. The final predicted fatigue life is $N_{f}^{P D}=40850$ (cycles), meanwhile, the experimental value is $N_{f}^{\text {experimental }}=42600$ (cycles).

Therefore, the relative error in terms of the final fatigue life can be estimated as

$\operatorname{error}(\%)=\frac{N_{f}^{P D}-N_{f}^{\text {ex perimental }}}{N_{f}^{\text {ex perimental }}} \times 100=-4.108 \%$

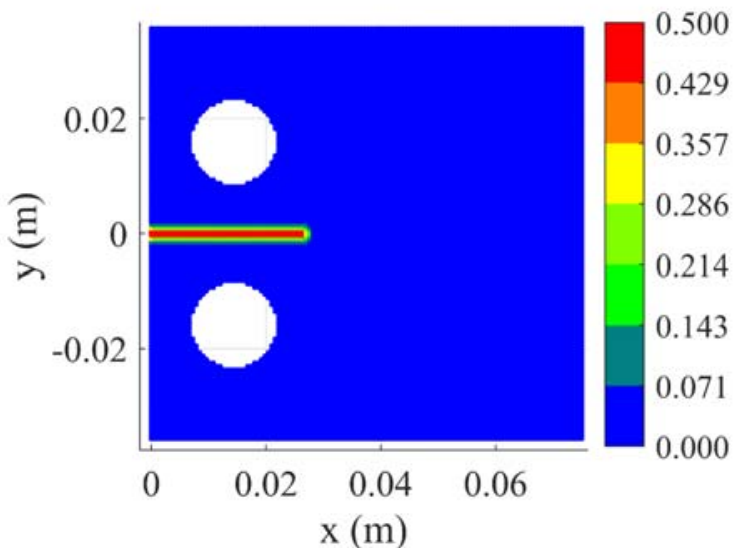

(a)

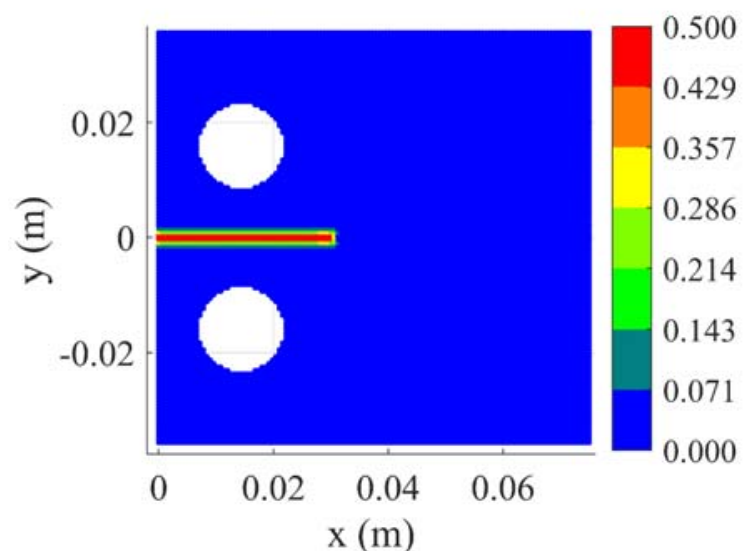

(b) 


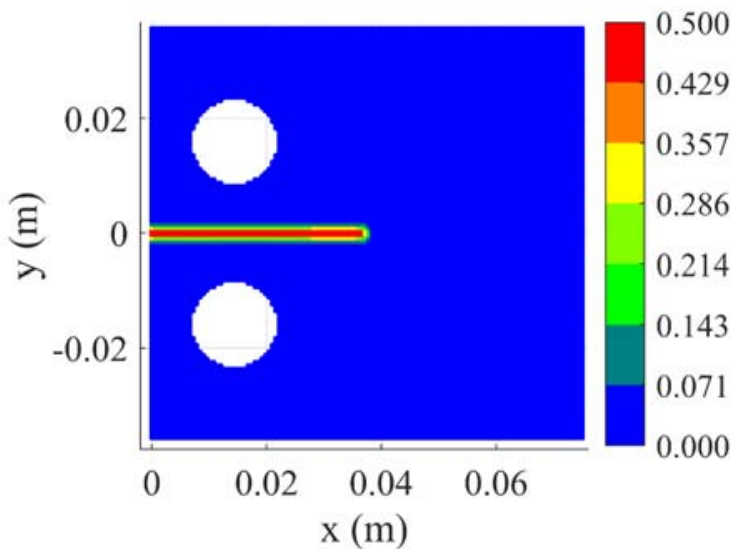

(c)

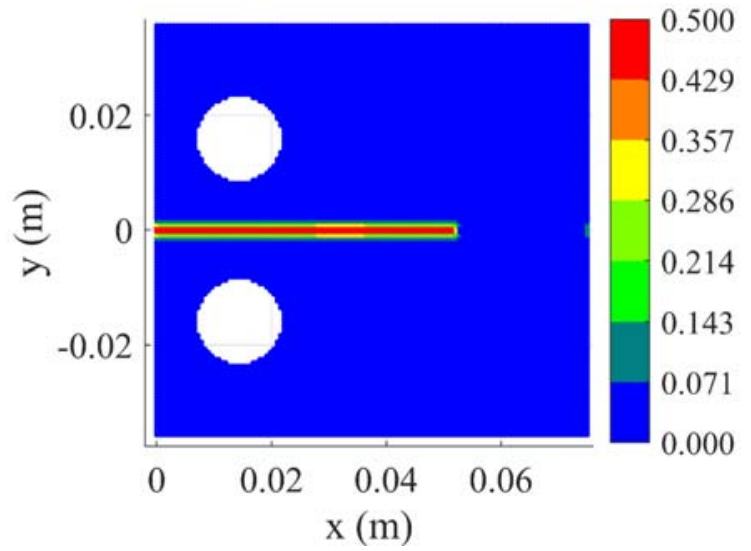

(d)

Fig. 11. Fatigue damage evolution at (a) 2000 cycles, (b) 15000 cycles, (c) 30000 cycles, (d) 40850 cycles

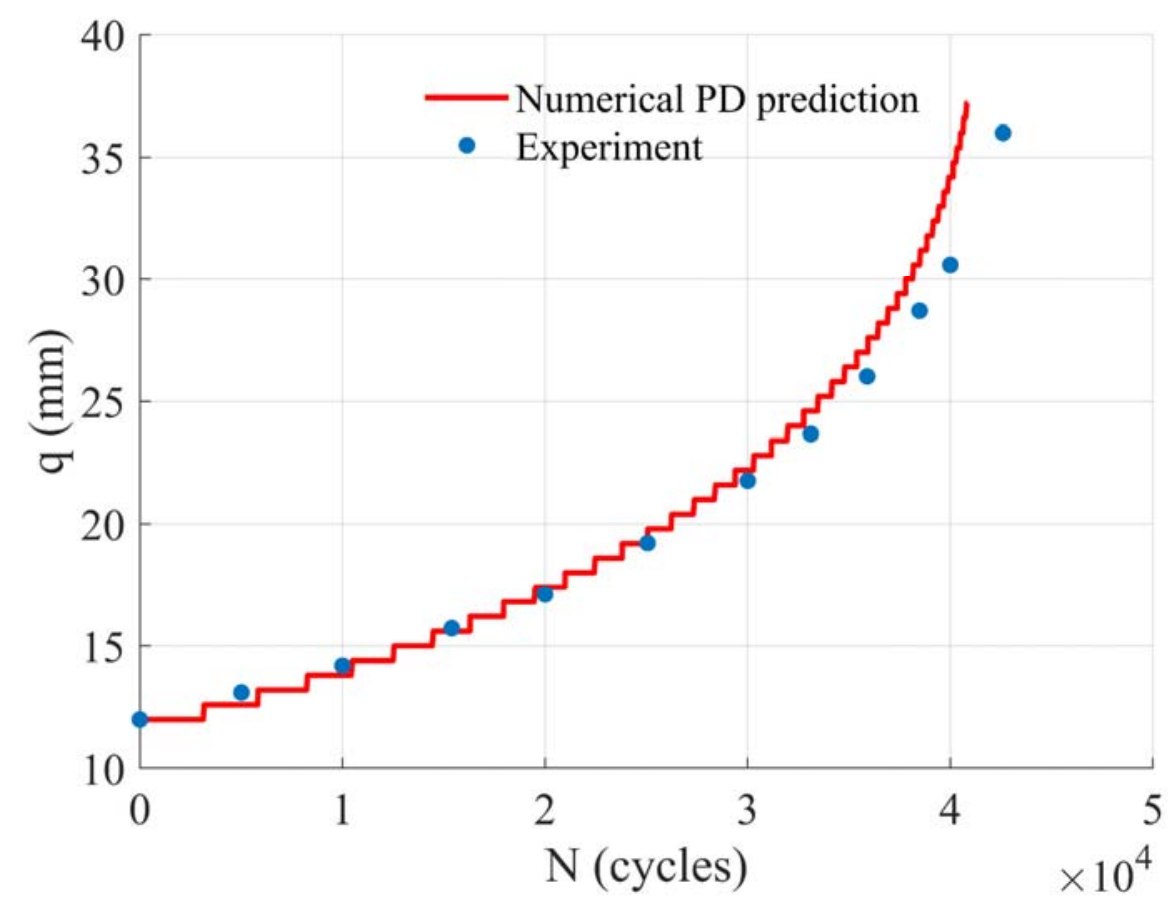

Fig. 12. Fatigue crack length, $q$ versus load cycle, $N$ (the experimental data is obtained from [70])

\subsection{Mixed-mode fatigue crack propagation}

After verifying the energy-based PD fatigue model for mode-I fatigue crack evolution, the proposed PD model is further used to predict fatigue damages in the mixed-mode loading conditions as shown in Fig. 13. The dimensions for the specimen are in $\mathrm{mm}$ as shown in Fig. 13(b). The plate has an initial notch with a length of $40 \mathrm{~mm}$ and a $5 \mathrm{~mm}$ initial fatigue crack is created [70]. The material properties are the same as in Section 6.1. The loading is defined by the extreme load $P=16 \mathrm{kN}$, load ratio $R=0.1$ and the loading angle $\alpha$ as shown in Fig. 13(a) and Fig. 14(a).

The boundary and loading conditions for the numerical models suggested by Sajith, et al. [70] are shown in Fig. 14(a). In PD, the model is uniformly discretized with a mesh size of $\Delta x=6 \times 10^{-4} \mathrm{~mm}$. As shown in Fig. 14(b), material points, shown in blue, green, black, and red, which are associated with 6 cut-outs, are defined as rigid with the elastic modulus of 
$E_{\text {rigid }}=200 E$. Material points, shown in black in Fig. 14(b), located at the centers of the lower cut-outs are fixed. Meanwhile, material points, shown in red in Fig. 14(b), located at the centers of the upper cut-outs are subjected to applied forces as [70]

$$
\begin{aligned}
& F_{1}=P(0.5 \cos \alpha+(e / f) \sin \alpha) \\
& F_{2}=-P \sin \alpha \\
& F_{3}=P(0.5 \cos \alpha-(e / f) \sin \alpha)
\end{aligned}
$$

Dimensions are in $\mathrm{mm}$

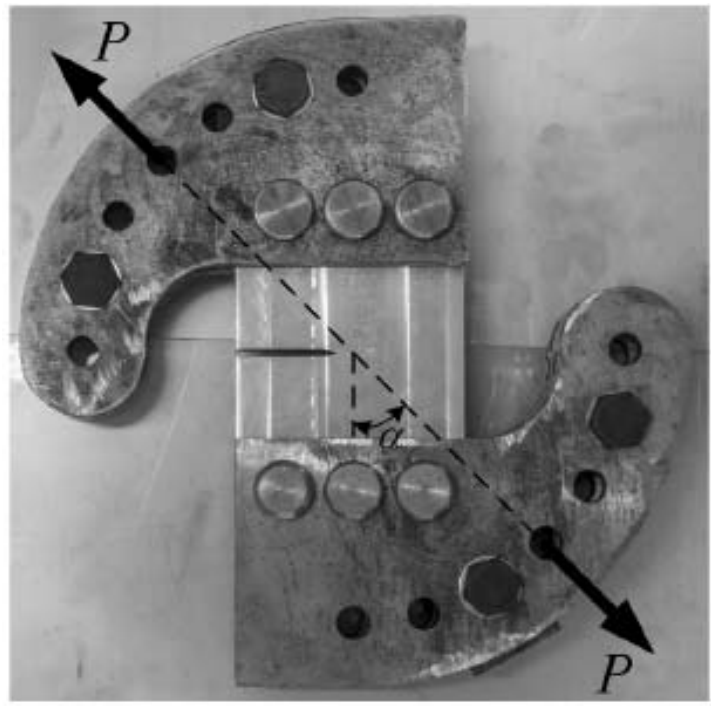

(a)

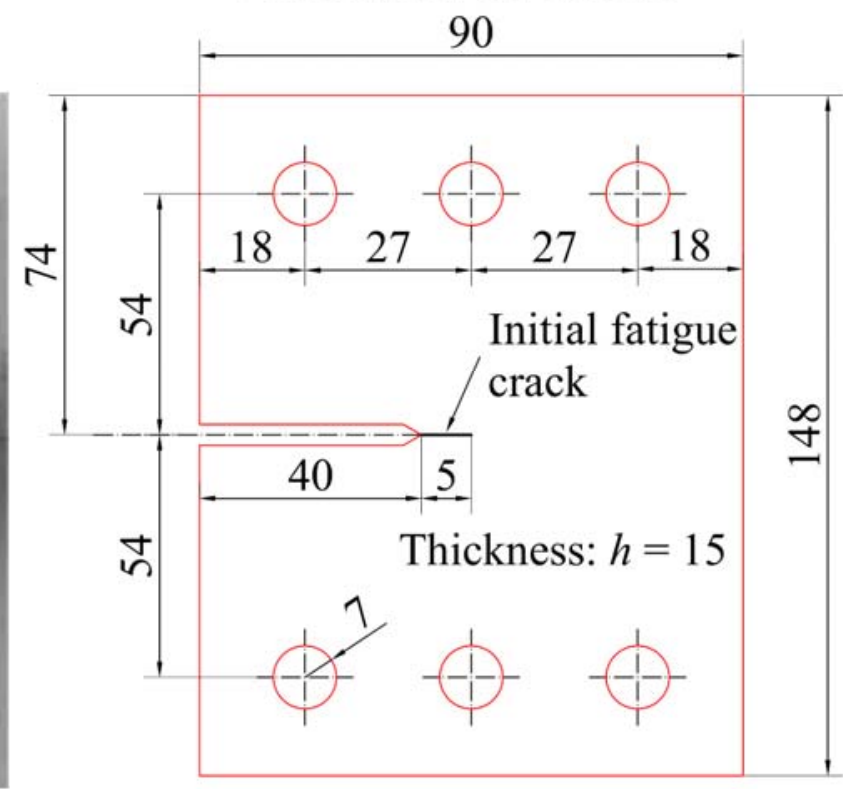

(b)

Fig. 13. Mixed-mode fatigue problem (a) experimental configuration in [70], (b) specimen's dimensions

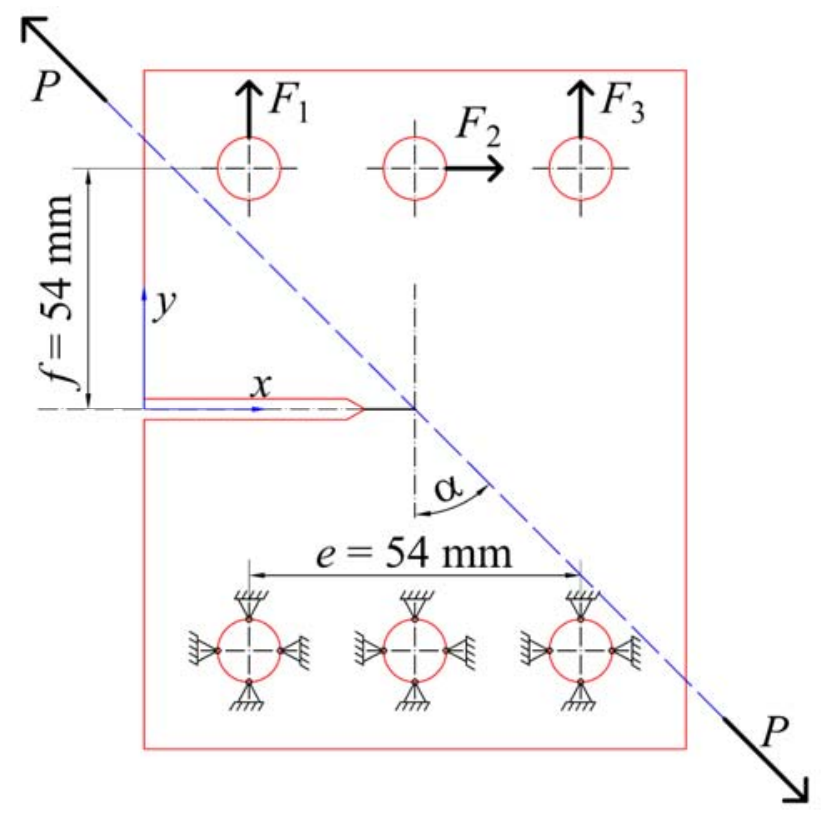

(a)

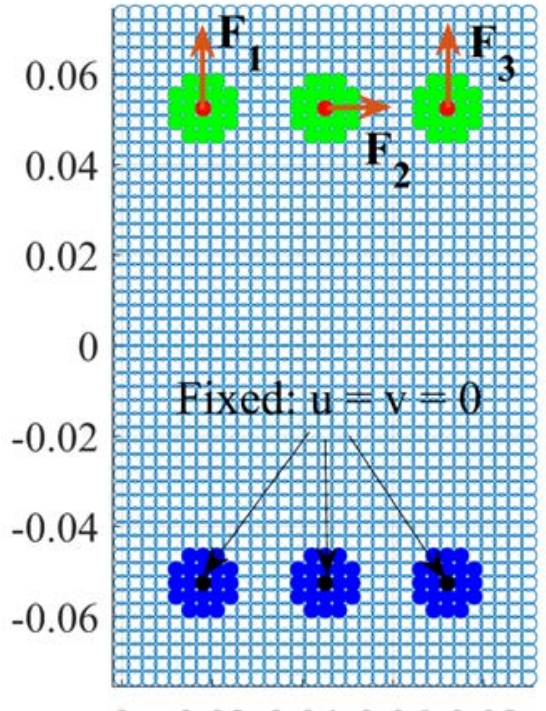

$\begin{array}{lllll}0 & 0.02 & 0.04 & 0.06 & 0.08\end{array}$

(b)

Fig. 14. Loading and boundary conditions (a) suggested by Sajith, et al. [70], (b) used in the PD model 


\subsubsection{Crack path prediction}

In peridynamics, the horizon sizes and mesh sizes can have significant effects on the crack propagation, especially for mixed-mode loading problems. Therefore, to find the best suitable mesh size and horizon size for mixed-mode loading problems, the fatigue damage in the plate subjected to mixed-mode loading condition with $\alpha=45^{\circ}$ is predicted by using the developed PD model with different mesh sizes and horizon sizes. Since the value of fatigue parameter $B_{2(k)(j)}$ only affects the results for the $q-N$ curve, all the PD simulations (with different horizon sizes, different mesh sizes) use the same values of fatigue parameters calibrated from the previous example.

Fig. 15 shows the fatigue crack paths predicted by the PD model with different horizon sizes, $\delta=2 \Delta x, 3 \Delta x, 4 \Delta x$, for the mixed-mode loading with $\alpha=45^{\circ}$. In these PD simulations, the same mesh size used in mode I fatigue problem, which is $\Delta x=6 \times 10^{-4} \mathrm{~m}$, is used. As shown in Fig. 15(a), the crack path predicted by the PD model with the horizon size of $\delta=2 \Delta x$ is nearly straight which is incorrect when compared to the experimental result [62]. On the other hand, the crack paths predicted by the PD model with horizon sizes of $\delta=3 \Delta x$ and $\delta=4 \Delta x$ are nearly identical as shown in Fig. 15(b, c, d). Moreover, the predicted crack paths in these two cases, $\delta=3 \Delta x$ and $\delta=4 \Delta x$, match well with the crack path in the experiment [62]. Therefore, to reduce the computational cost, the horizon size $\delta=3 \Delta x$ can be used.

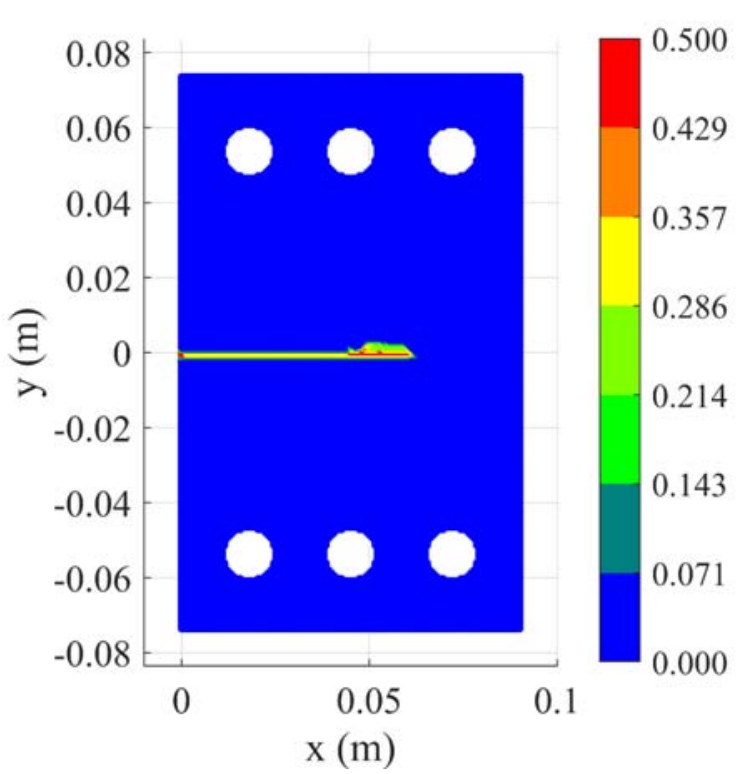

(a)

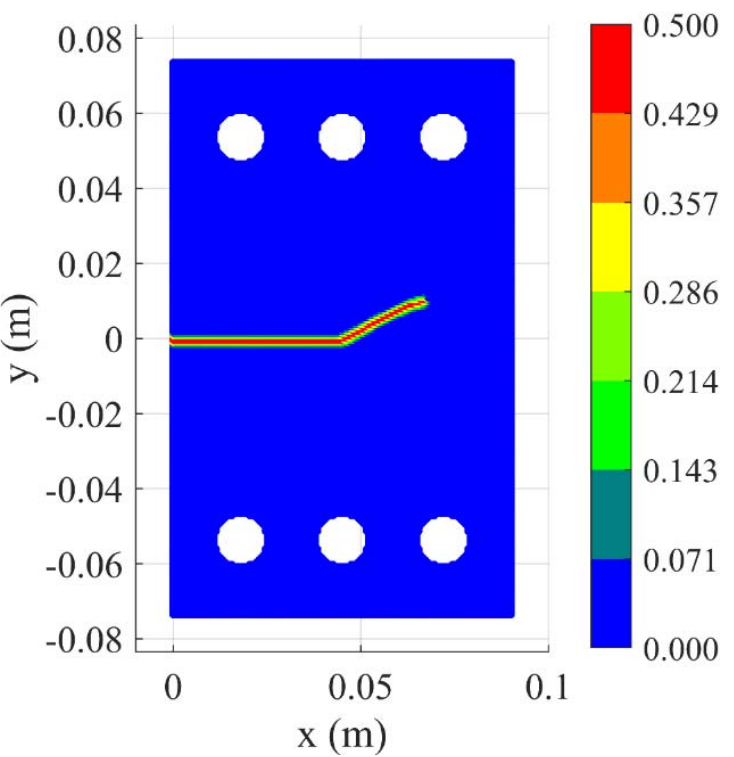

(b) 


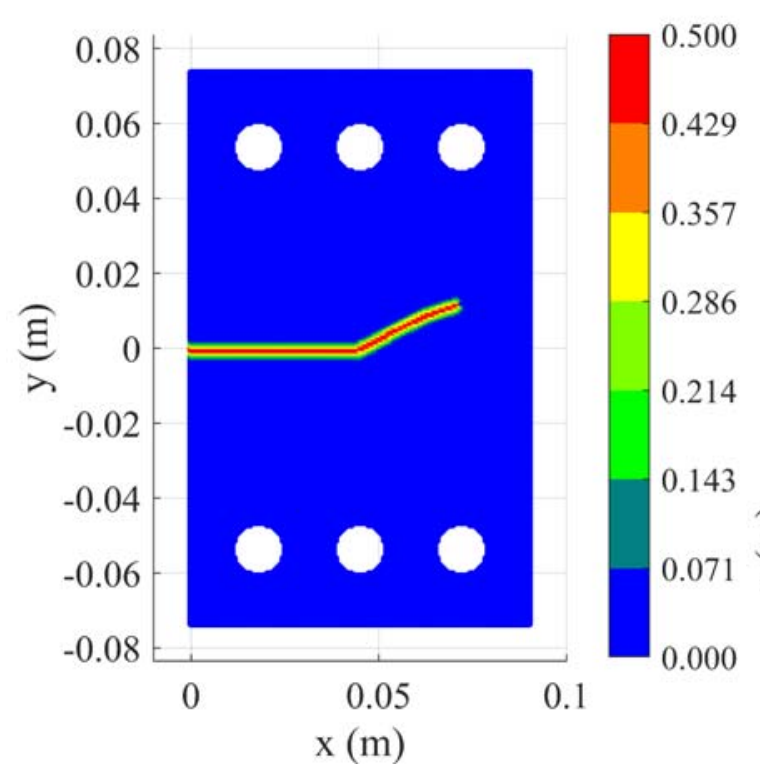

(c)

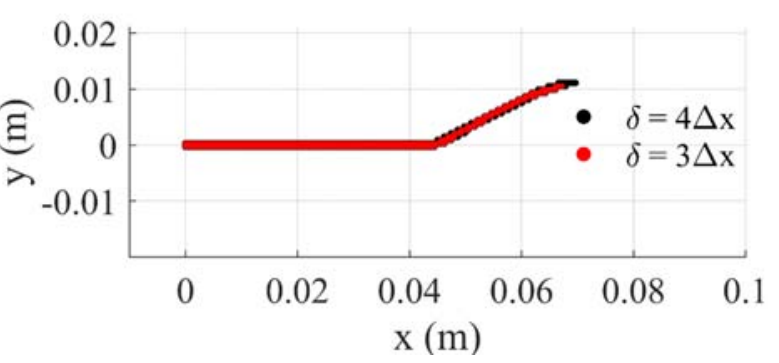

(d)

Fig. 15. Fatigue crack path in mixed-mode loading with $\alpha=45^{\circ}$ predicted by the PD model with different horizon sizes (a) $\delta=2 \Delta x$ (b) $\delta=3 \Delta x$, (c) $\delta=4 \Delta x$, (d) a comparison between two cases $\delta=3 \Delta x$ and $\delta=4 \Delta x$ (the mesh size of $\Delta x=6 \times 10^{-4} \mathrm{~m}$ is used for all cases)

Fig. 16 shows the fatigue crack paths predicted by the PD model with different mesh sizes, $\Delta x=18 \times 10^{-4} \mathrm{~m}, 9 \times 10^{-4} \mathrm{~m}, 6 \times 10^{-4} \mathrm{~m}, 4.5 \times 10^{-4} \mathrm{~m}$ for the mixed-mode loading with $\alpha=45^{\circ}$. In these PD simulations, the horizon size $\delta=3 \Delta x$ is used. As shown in Fig. 16, all the crack paths predicted by the PD fatigue model with different mesh sizes match the experimental results [62]. Moreover, the crack paths predicted by the PD model with mesh sizes of $\Delta x=6 \times 10^{-4} \mathrm{~m}$ and $\Delta x=4.5 \times 10^{-4} \mathrm{~m}$ are nearly identical as shown in Fig. 16(c, d). Therefore, to reduce the computational cost, the mesh size of $6 \times 10^{-4} \mathrm{~m}$ can be used.

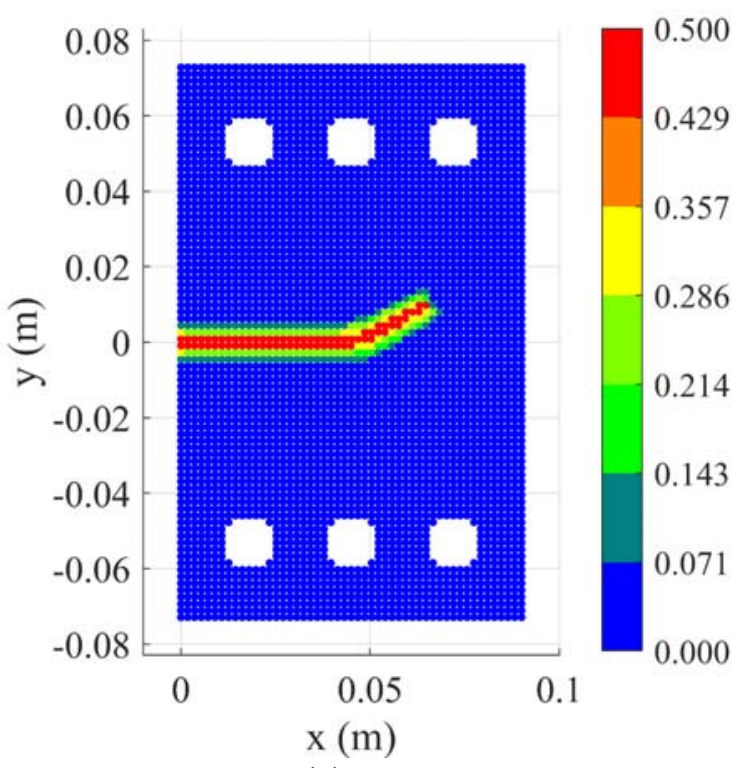

(a)

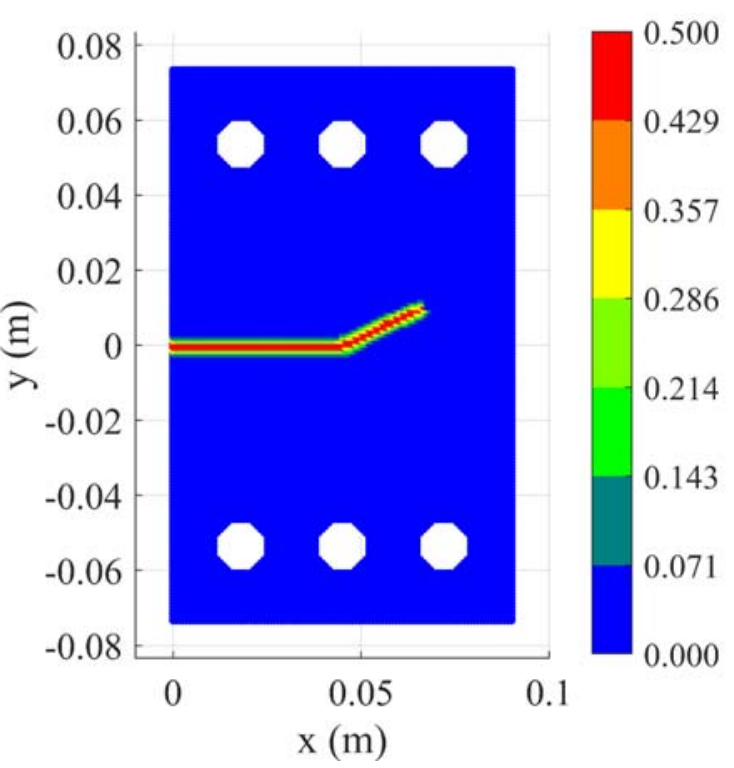

(b) 


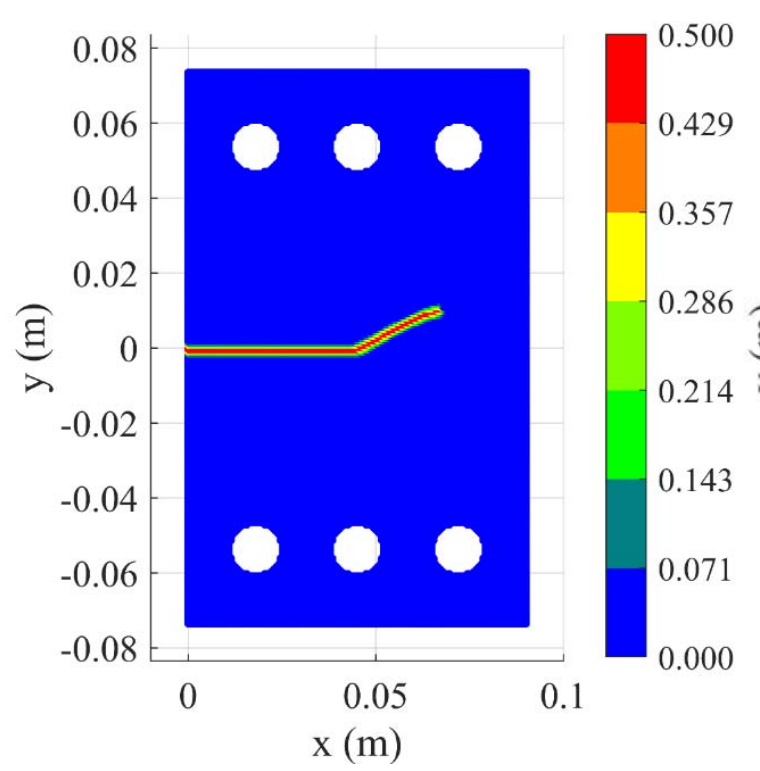

(c)

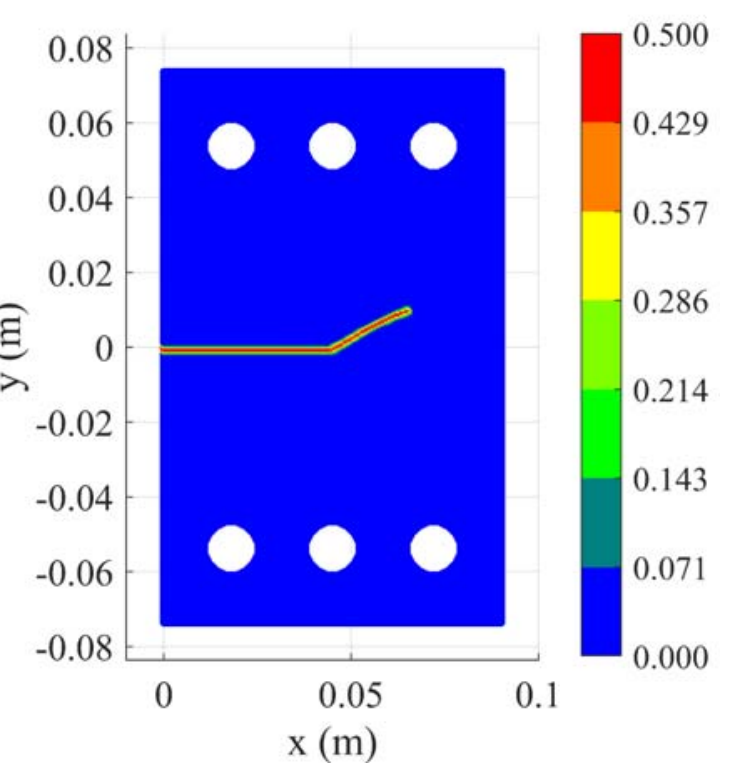

(d)

Fig. 16. Fatigue crack path in mixed-mode loading with $\alpha=45^{\circ}$ predicted by the PD model with different mesh sizes (a) $\Delta x=18 \times 10^{-4} \mathrm{~m}$ (b) $\Delta x=9 \times 10^{-4} \mathrm{~m}$, (c) $\Delta x=6 \times 10^{-4} \mathrm{~m}$, (d) $\Delta x=4.5 \times 10^{-4} \mathrm{~m}$ (the horizon size $\delta=3 \Delta x$ is used for all cases)

Note that, the values of $A_{2}$ and $B_{2(k)(j)}$ do not affect the predicted results in terms of crack paths. Therefore, for the convergence study of the mesh size, an arbitrary value of $A_{2}$ can be used, and $B_{2(k)(j)}$ can obtained from $A_{2}$ by using Eq. (46b). Later, when the suitable mesh size is chosen, the correct values for $A_{2}$ and $B_{2(k)(j)}$ can be calibrated by using the chosen mesh size.

Fig. 17 shows the fatigue crack propagation in the mixed-mode loading in the case of $\alpha=45^{\circ}$ predicted by the PD model with the mesh size of $\Delta x=6 \times 10^{-4} \mathrm{~m}$ and the horizon size of $\delta=3 \Delta x$. As shown in Fig. 17(a), the crack starts propagating upward at 10000 loading cycles. The angle of crack propagation with respect to the horizontal axis is measured numerically as $\beta_{P D}=38.66^{\circ}$ at 30000 loading cycles as shown in Fig. 17(c). This observation has good agreement with the experimental results which is $\beta_{\text {experiment }}=40.263^{\circ}$. Later, the crack propagates with a slightly smaller angle and reaches the final fatigue crack growth state (prior to phase III) at 42768 loading cycles as shown in Fig. 17(d). The slight reduction of crack propagation angles is also observed in the experiment by Sajith, et al. [70], Chung and Yang [73], Borrego, et al. [74]. 


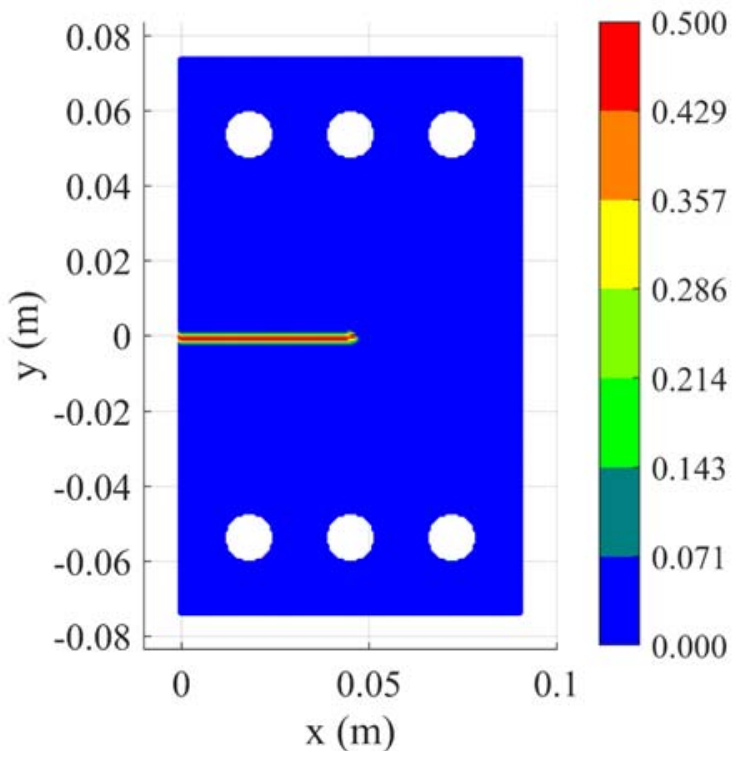

(a)

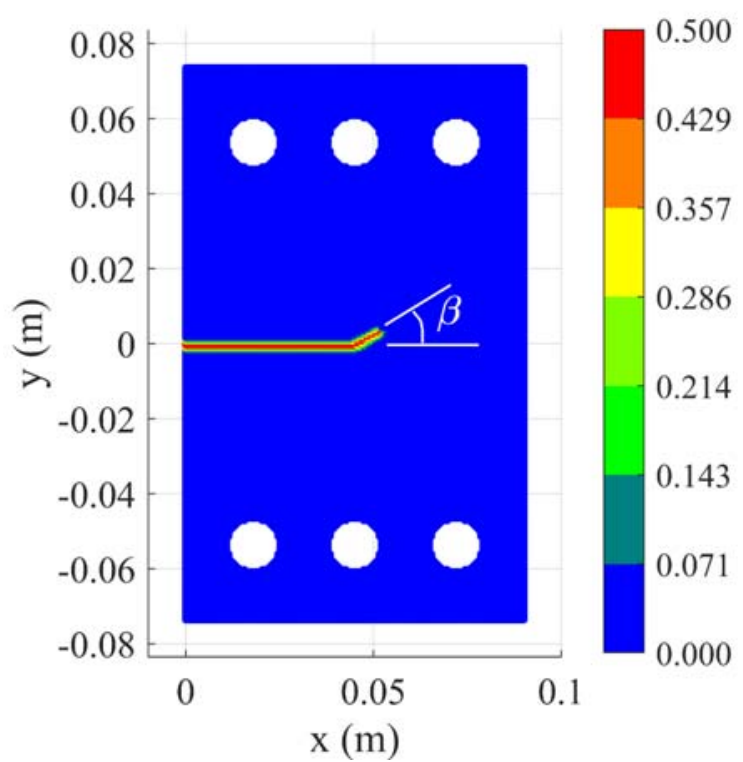

(c)

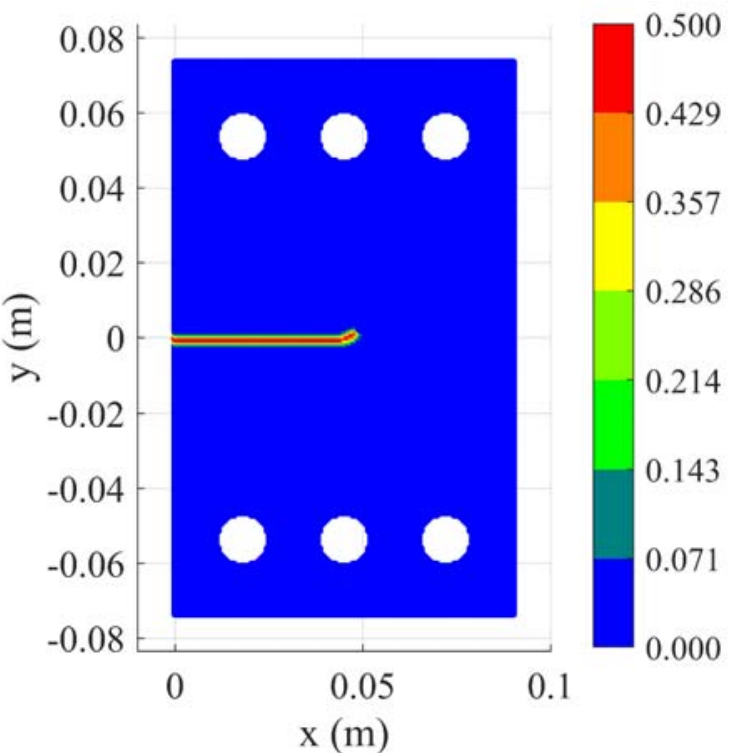

(b)

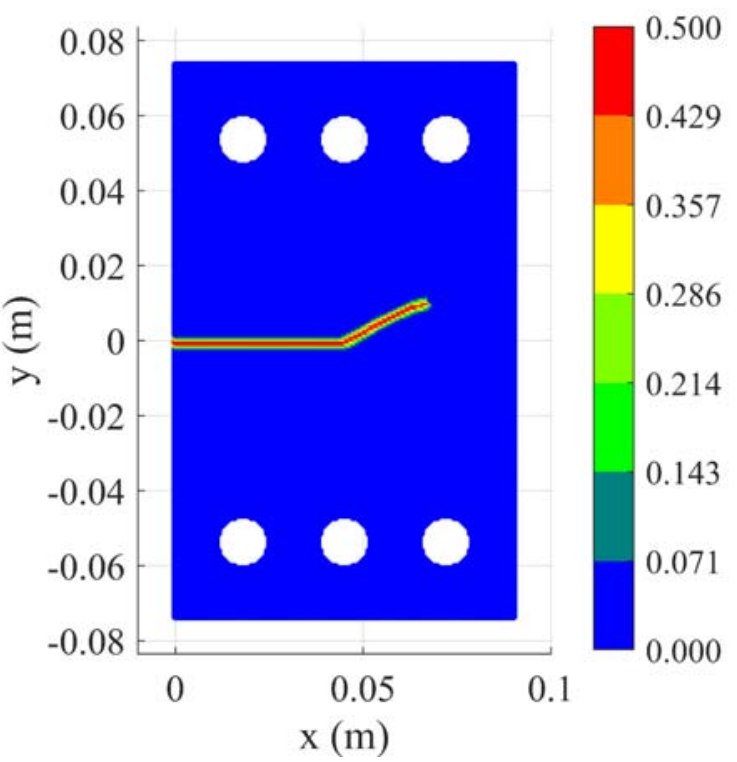

(d)

Fig. 17. Fatigue crack evolution in mixed-mode loading with $\alpha=45^{\circ}$ at (a) 10000 (b) 20000 , (c) 30000 , (d) 42768 cycles

Fig. 18 shows the prediction results with the mesh size of $\Delta x=6 \times 10^{-4} \mathrm{~m}$ and the horizon size of $\delta=3 \Delta x$ for the mixed-mode fatigue crack propagation in the case of $\alpha=60^{\circ}$. Fig. 18(a-c) shows the crack evolution at 20000,35000 and 44500 loading cycles, respectively. Similar to the previous loading condition, the fatigue crack also propagates upward but with a larger angle $\beta_{P D}=50.19^{\circ}$. This observation has very good agreement with the experimental results $\beta_{\text {experiment }}=51.33^{\circ}[70]$. Similar to the previous loading condition, after 44500 loading cycles, the crack starts propagating with a slightly smaller angle and reaches the final fatigue crack growth state (prior to phase III) at 53727 loading cycles as shown in Fig. 18(d). This observation has good agreement with the experimental results studied by Sajith, et al. [70], Chung and Yang [73], Borrego, et al. [74]. 


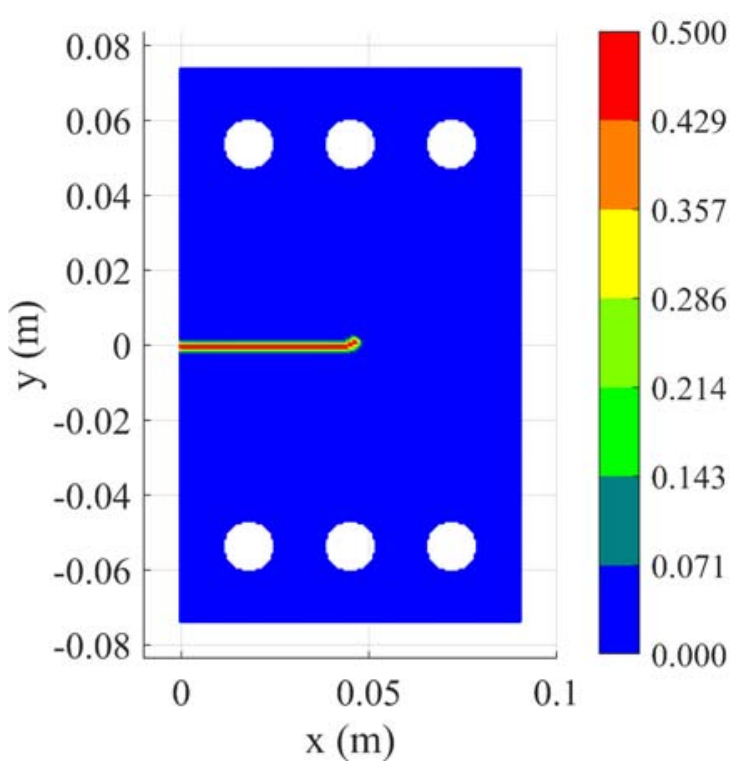

(a)

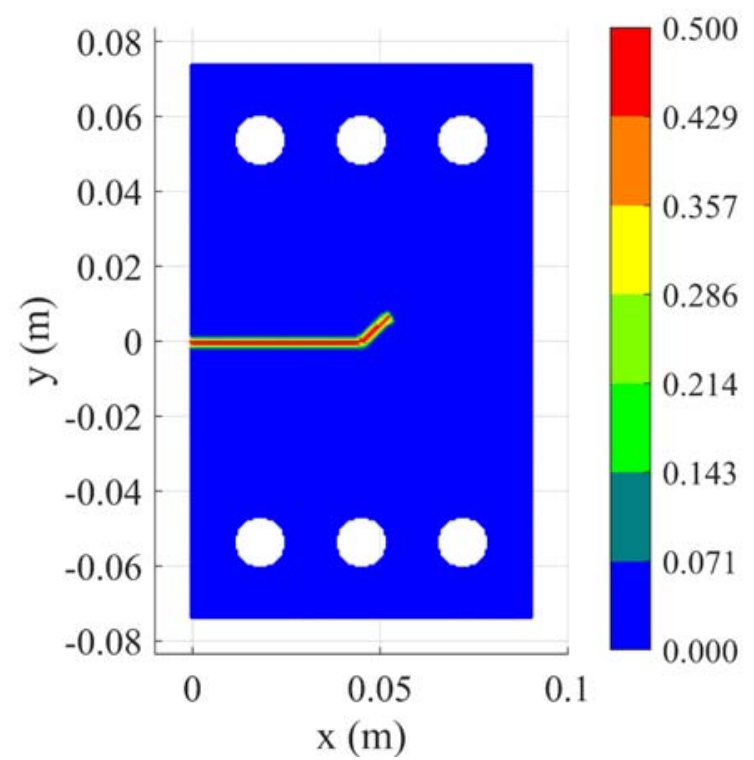

(c)

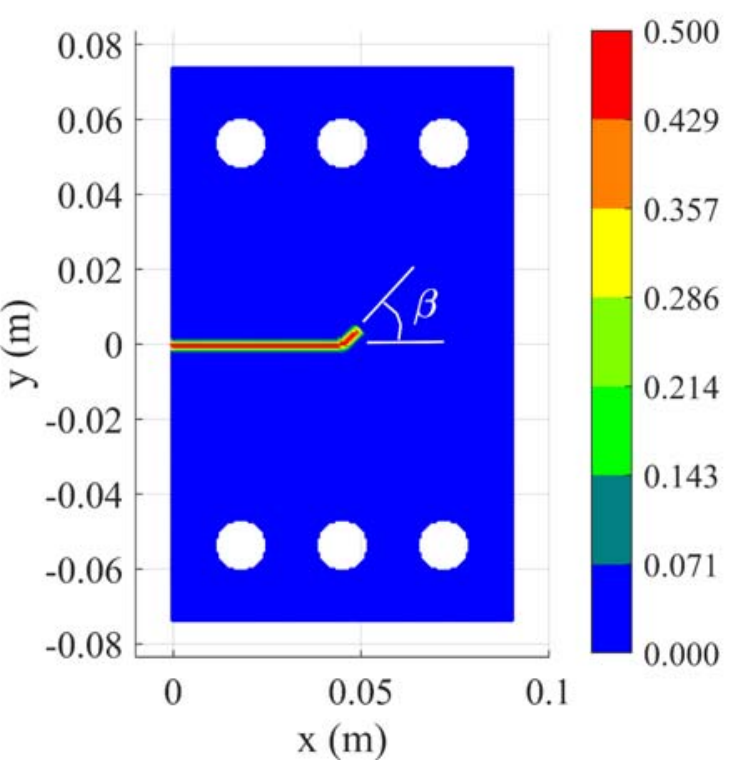

(b)

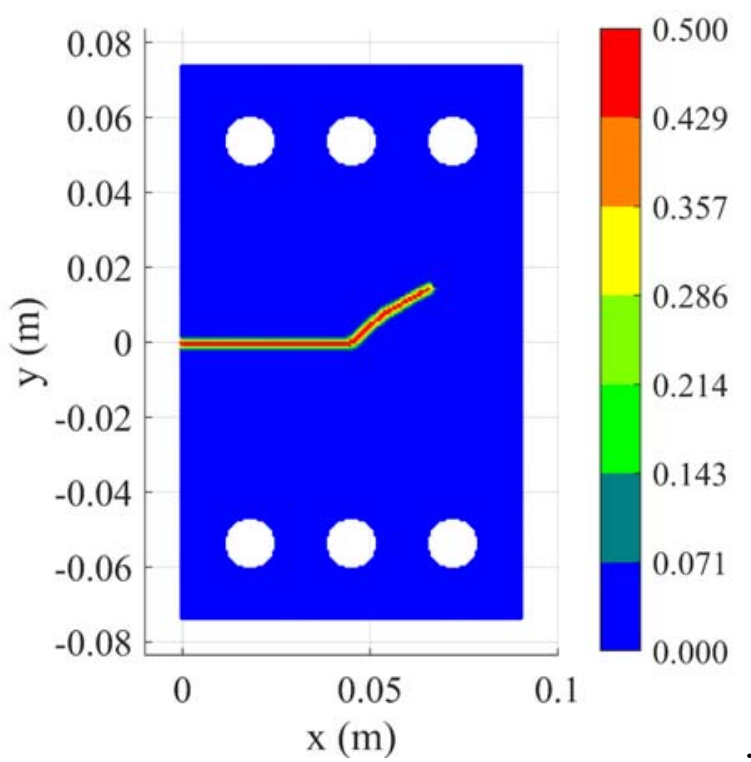

(d)

Fig. 18. Fatigue crack evolution in mixed-mode loading with $\alpha=60^{\circ}$ at (a) 20000 (b) 35000 , (c) 44500 , (d) 53727 cycles

Fig. 19 shows the crack tip positions in the two loading conditions predicted by the proposed energy-based PD fatigue model. As can be seen from the figure, the PD results have good agreement with the experimental results studied by Sajith, et al. [70] which shows the accuracy of the proposed PD model in terms of crack path prediction. 


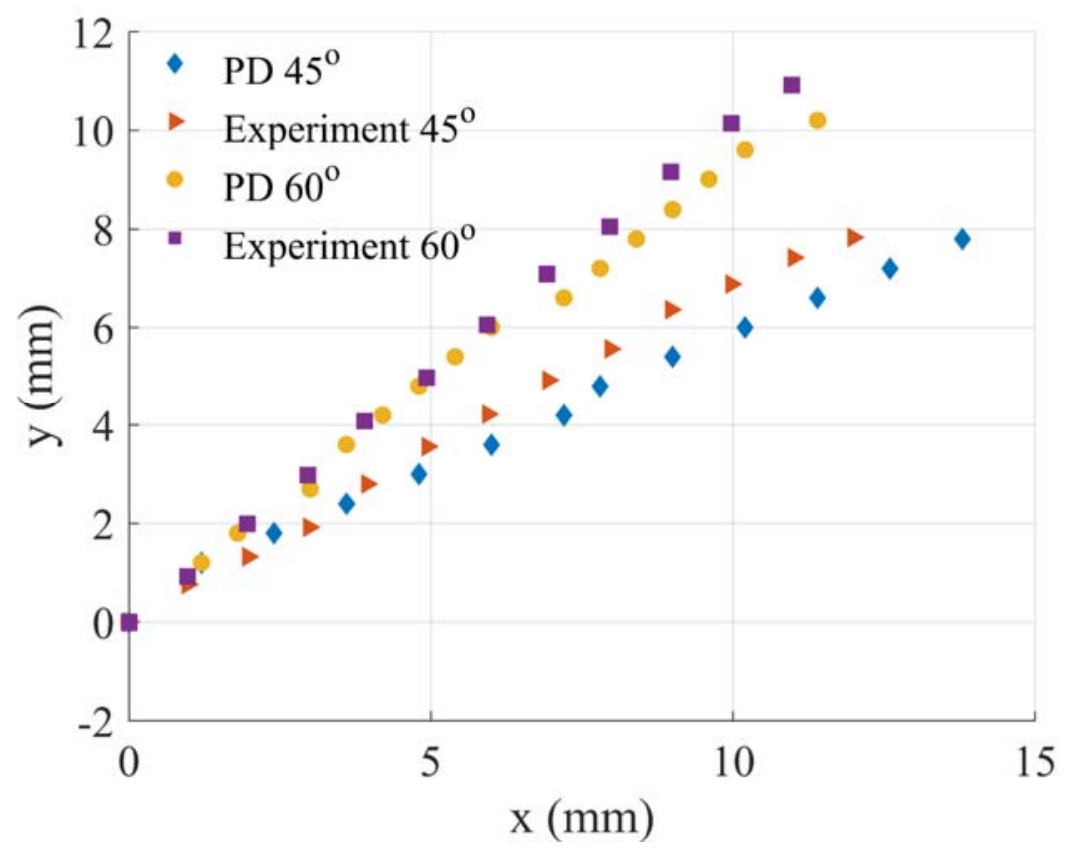

Fig. 19. Crack tip locations (the experimental data is obtained from [70])

\subsubsection{Fatigue life prediction}

Fig. 20 shows the fatigue crack length, $q$ versus load cycle, $N$ in two loading conditions. As can be seen from Fig. 20(a) for the loading condition with $\alpha=45^{\circ}$, the predicted results have very good agreement with experimental results in the first 28000 loading cycles. Later, there is a small difference between the two results. The predicted results show a slightly slower crack growth compared to the observation from the experiment [70]. As can be seen from Fig. 20(b), the predicted crack growth in case of $\alpha=60^{\circ}$ has very good agreement with the experimental results which shows the accuracy of the proposed PD fatigue model in terms of fatigue life prediction.

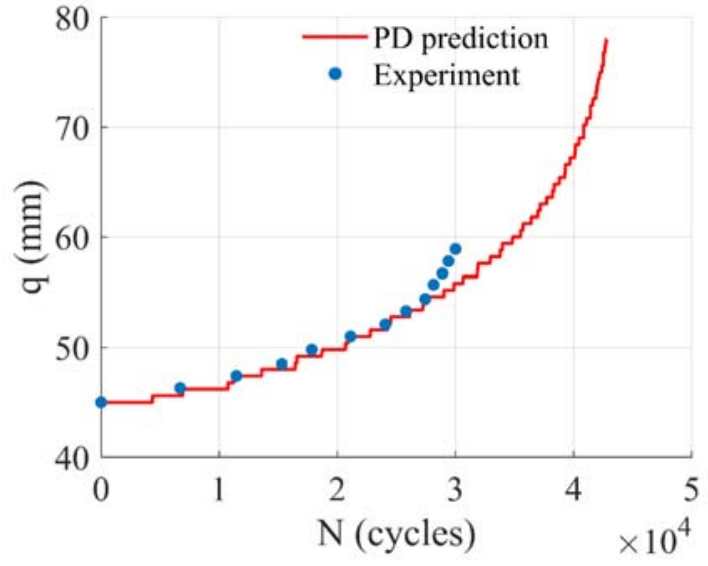

(a)

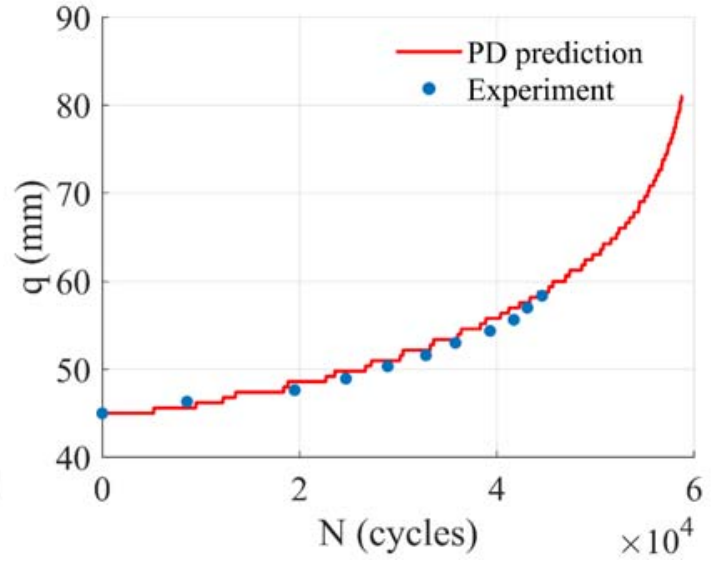

(b)

Fig. 20. Crack length, $q$ versus load cycle, $N$ for mixed-mode fatigue crack growth with load angle (a) $\alpha=45^{\circ}$, (b) $\alpha=60^{\circ}$ (the experimental data is obtained from [70])

\section{Conclusion}

In this study, a novel energy-based PD model for fatigue damage prediction is proposed. The definition of cyclic bond energy release rate range is proposed and used for fatigue equations 
for the first time in the PD literature. The numerical procedure to predict fatigue cracking by using the proposed PD model is presented.

The capability of the proposed PD model is verified by considering mode-I and mixed-mode fatigue crack propagations. The results predicted by the proposed PD model show good agreements with the experimental results in terms of the crack paths as well as the crack growth rates.

The developed PD model can be further extended to the 3D formulation and it can also be used to predict fatigue damage for structures during the operation process.

\section{Acknowledgement}

The authors are grateful for the support of the Ministry of Education and Training in Vietnam and the University of Strathclyde. The results were obtained using the ARCHIE-WeSt HighPerformance Computer (www.archie-west.ac.uk) based at the University of Strathclyde. 


\section{Appendix A. Calibration for parameter $A_{2}$}

In this section, details of the calibration for the parameter $A_{2}$ used in Section 6.2.1 are presented. As presented in Section 3.2 and Section 6.1, the calibration for the parameter $A_{2}$ is conducted by the following steps;

Step 1: Assume a trial value for $A_{2}$ as: $A_{2(\text { trial })}=1174$

Step 2: Conduct a PD fatigue simulation with the trial value $A_{2(\text { trial })}=1174$ and calculate $(d q / d N)_{\text {(trial) }}$ and $\Delta K_{\text {(trial) }}$.

Step 2.1. Calculate $(d q / d N)_{(\text {trial })}$

First, by using the PD results for $A_{2(\text { trial }}=1174$, the crack length, $q_{\text {(trial) }}$ versus load cycle, $N_{\text {(trial) }}$ is obtained as shown in red in Fig. 21. Later, a smoothed curve for the PD results is obtained as shown in blue in Fig. 21. Finally, $(d q / d N)_{\text {(trial) }}$ is numerically obtained from the smoothed curve as

$$
(d q / d N)_{\text {(trial) }}=\frac{\Delta q_{(\text {trial })}}{\Delta N_{(\text {trial }}}
$$

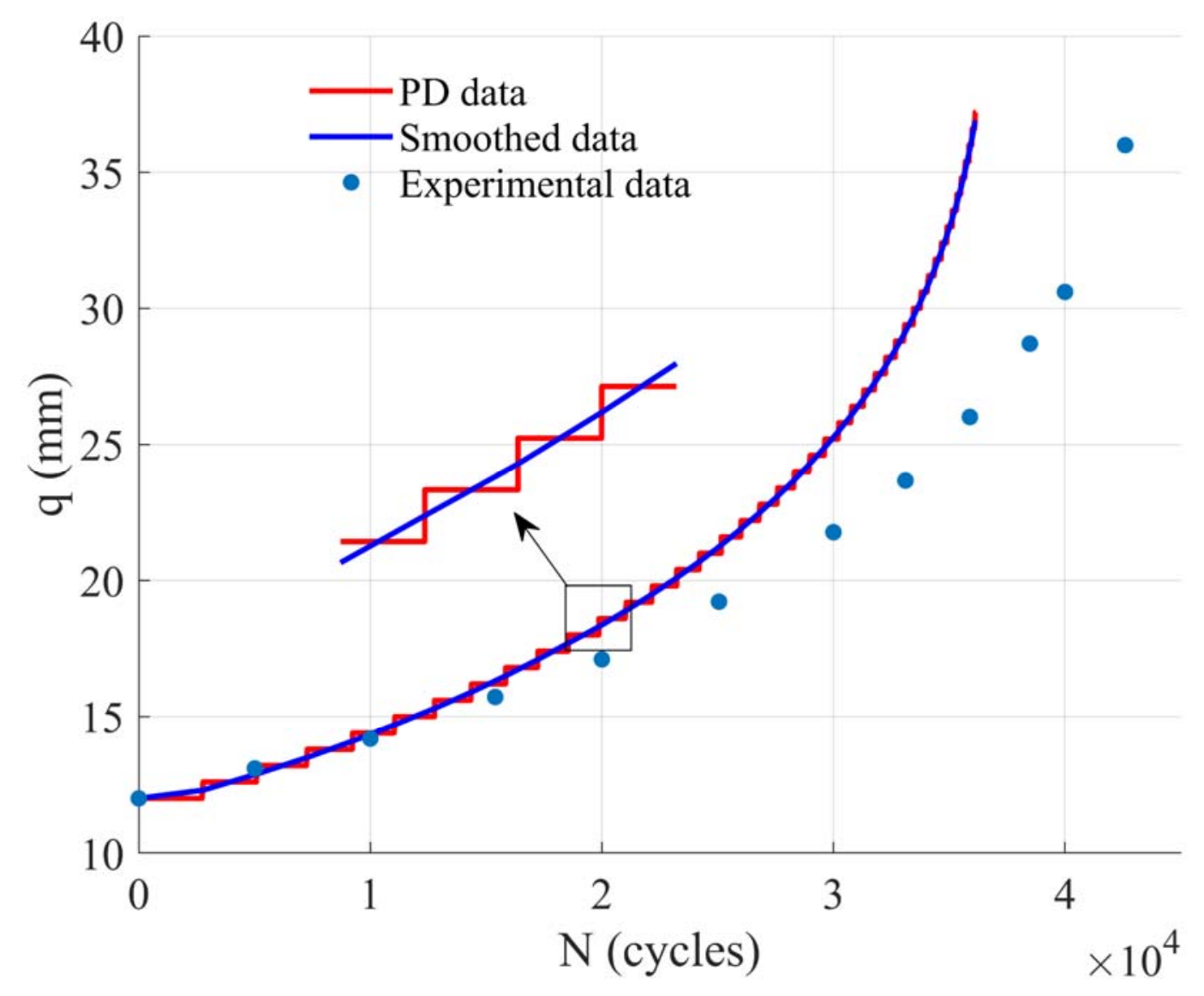

Fig. 21. Fatigue crack length, $q_{\text {(trial) }}$ versus load cycle, $N_{\text {(trial) }}$ (the experimental data is obtained from [70]) 
Step 2.2. Calculate $\Delta K_{\text {(trial) }}$

By using the data of the crack length, $q_{\text {(trial) }}$ obtained from the trial PD fatigue simulation and by assuming that the material is linear-elastic, the stress intensity factor (SIF) range, $\Delta K_{(\text {trial })}$ can be calculated as [75]

$$
\Delta K_{\text {(trial) }}=\frac{\Delta P}{h \sqrt{W}} \frac{2+\bar{q}}{(1-\bar{q})^{3 / 2}}\left(0.886+4.64 \bar{q}-13.32 \bar{q}^{2}+14.72 \bar{q}^{3}-5.6 \bar{q}^{4}\right)
$$

with

$$
\begin{aligned}
& \bar{q}=\frac{q_{(\text {trial })}}{W} \\
& \Delta P=P_{\text {max }}(1-R)
\end{aligned}
$$

Step 3: Plot the scatter data of $(d q / d N)_{\text {(trial) }}$ versus $\Delta K_{\text {(trial) }}$ in the logarithmic scale and find the best fit Paris law equation for the scatter data: $(d q / d N)_{\text {(trial) }}=C_{\text {(trial) }} \Delta K^{M}$ First, by using the $(d q / d N)_{\text {(trial) }}$ and $\Delta K_{\text {(trial) }}$ calculated in Eq. (A1) and Eq. (A2a), respectively; the scatter data $(d q / d N)_{\text {(trial) }}$ versus $(\Delta K)_{\text {(trial) }}$, shown in blue in Fig. 22, is plotted in the logarithmic scale. From this scatter data, the best fit curve, shown in black in Fig. 22, is obtained as

$$
(d q / d N)_{\text {(trial) }}=4.8295 \times 10^{-7} \Delta K^{2.6183}
$$

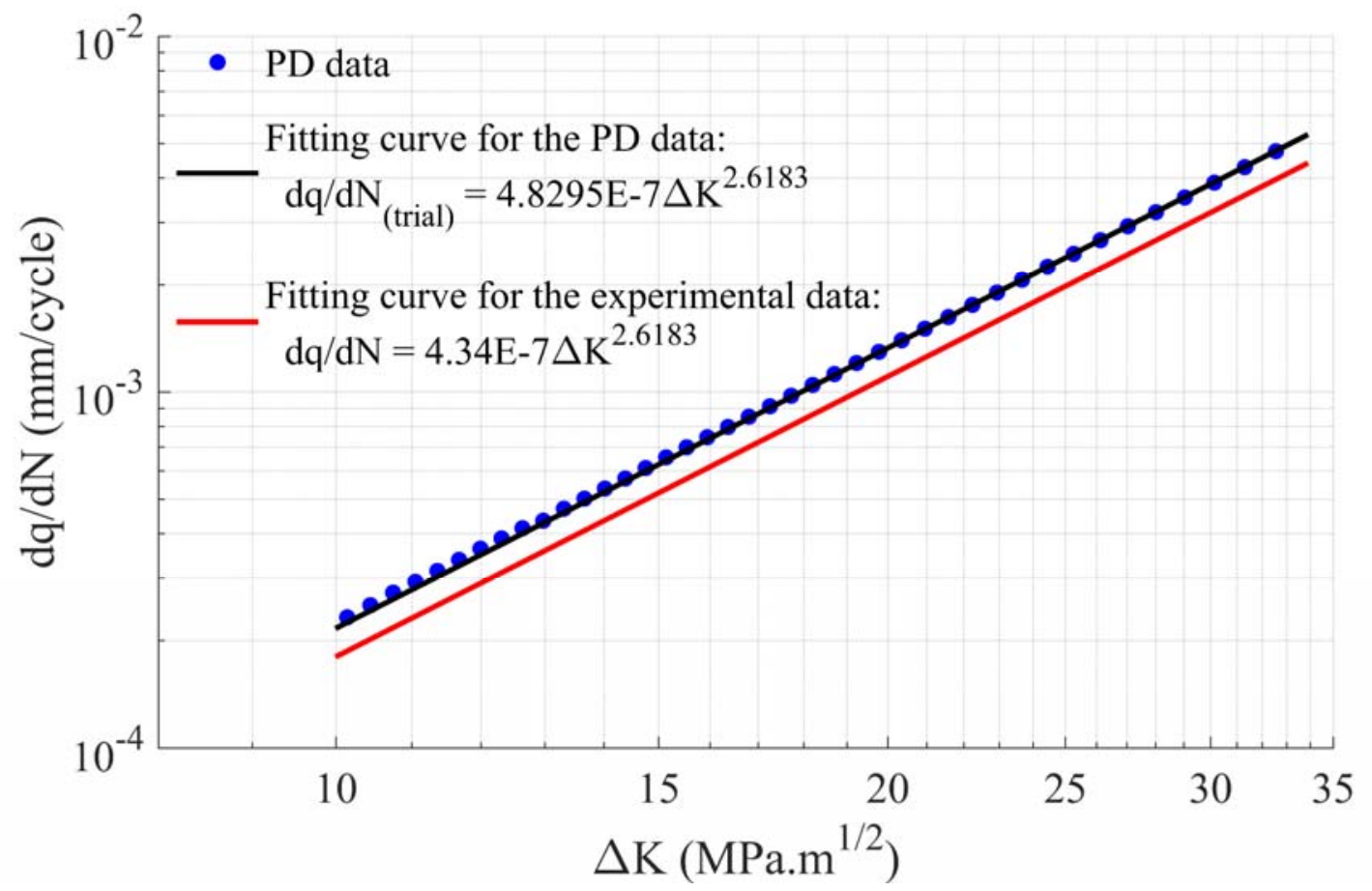

Fig. 22. Fatigue crack growth curve for $(d q / d N)_{\text {(trial) }}$ versus $(\Delta K)_{\text {(trial) }}$ with $A_{2(\text { (trial) }}=1174$ (the experimental data is obtained from [70]) 
Step 4: Calibrate the value for $A_{2}$

As it can be found in [70], the best fit Paris law equation for the experimental results is written as

$(d q / d N)_{\text {(experiment) }}=4.34 \times 10^{-7} \Delta K^{2.6183}$

Therefore, the value of the parameter $A_{2}$ can be estimated from Eq. (26b) as

$A_{2}=A_{2 \text { (trial) }} \frac{4.34 \times 10^{-7} \Delta K^{2.6183}}{4.8295 \times 10^{-7} \Delta K^{2.6183}}=1174 \frac{4.34 \times 10^{-7}}{4.8295 \times 10^{-7}}=1055$

\section{Appendix B. Implicit solver for static conditions in peridynamics}

In this section, the implicit solver for ordinary state-based peridynamics for static conditions is presented. First, the PD equations of motion for static conditions given in Eq. (6) can be rewritten as

$$
\begin{aligned}
& -\sum_{j=1}^{N_{k}} \psi_{(k)(j)}\left[2 a d \frac{1}{\xi}\left(\vartheta_{(k)}+\vartheta_{(j)}\right)+4 b s_{(k)(j)}\right] \cos \varphi V_{(j)}=\bar{b}_{x(k)} \\
& -\sum_{j=1}^{N_{k}} \psi_{(k)(j)}\left[2 a d \frac{1}{\xi}\left(\vartheta_{(k)}+\vartheta_{(j)}\right)+4 b s_{(k)(j)}\right] \sin \varphi V_{(j)}=\bar{b}_{y(k)}
\end{aligned}
$$

By using the dilatation given in Eq. (3c) and the bond stretch given in Eq. (3d), the equations of motion given in Eq. (B1) can be rewritten in a matrix form as

$$
\begin{aligned}
& \sum_{j=1}^{N_{k}} \psi_{(k)(j)} \frac{2 a d}{\xi} V_{(j)}\left[\begin{array}{ll}
-\cos \varphi & -\cos \varphi \\
-\sin \varphi & -\sin \varphi
\end{array}\right]\left[\begin{array}{l}
\vartheta_{(k)} \\
\vartheta_{(j)}
\end{array}\right] \\
& +\sum_{j=1}^{N_{k}} \psi_{(k)(j)} \frac{4 b V_{(j)}}{\xi}\left[\begin{array}{cccc}
\cos ^{2} \varphi & -\cos ^{2} \varphi & \sin \varphi \cos \varphi & -\sin \varphi \cos \varphi \\
\sin \varphi \cos \varphi & -\sin \varphi \cos \varphi & \sin ^{2} \varphi & -\sin ^{2} \varphi
\end{array}\right]\left[\begin{array}{l}
u_{(k)} \\
u_{(j)} \\
v_{(k)} \\
v_{(j)}
\end{array}\right]=\left[\begin{array}{l}
\bar{b}_{x(k)} \\
\bar{b}_{y(k)}
\end{array}\right]
\end{aligned}
$$

or

$$
\sum_{j=1}^{N_{k}} \mathbf{k}_{(k)(j)}^{\text {dila }}\left[\begin{array}{l}
\vartheta_{(k)} \\
\vartheta_{(j)}
\end{array}\right]+\sum_{j=1}^{N} \mathbf{k}_{(k)(j)}^{B B}\left[\begin{array}{c}
u_{(k)} \\
u_{(j)} \\
v_{(k)} \\
v_{(j)}
\end{array}\right]=\left[\begin{array}{c}
\bar{b}_{x(k)} \\
\bar{b}_{y(k)}
\end{array}\right]
$$

with

$$
\mathbf{k}_{(k)(j)}^{B B}=\psi_{(k)(j)} \frac{4 b V_{(j)}}{\xi}\left[\begin{array}{cccc}
\cos ^{2} \varphi & -\cos ^{2} \varphi & \sin \varphi \cos \varphi & -\sin \varphi \cos \varphi \\
\sin \varphi \cos \varphi & -\sin \varphi \cos \varphi & \sin ^{2} \varphi & -\sin ^{2} \varphi
\end{array}\right]
$$

and

$$
\mathbf{k}_{(k)(j)}^{\text {dila }}=\psi_{(k)(j)} \frac{2 a d}{\xi} V_{(j)}\left[\begin{array}{ll}
-\cos \varphi & -\cos \varphi \\
-\sin \varphi & -\sin \varphi
\end{array}\right]
$$

where $\mathbf{k}_{(k)(j)}^{B B}$ represents the bond stiffness corresponding to the second term in the left-hand side of Eq. (B2a). The term $\mathbf{k}_{(k)(j)}^{\text {dila }}$ represents the bond stiffness corresponding to the first term in the left-hand side of Eq. (B2a). 
Note that, Eq. (B2) is the equation of motion for material point $k$. Assuming that the PD discretized model has $m$ material points. Therefore, the equation of motion for all material points in the PD model can be written as

$$
\left(\mathbf{K}^{\text {dila }}\right) \boldsymbol{\theta}+\left(\mathbf{K}^{B B}\right) \mathbf{U}=\overline{\mathbf{B}}
$$

with

$$
\begin{aligned}
& \mathbf{U}=\left[\begin{array}{llllllll}
u_{(1)} & u_{(2)} & \ldots & u_{(m)} & v_{(1)} & v_{(2)} & \ldots & v_{(m)}
\end{array}\right]^{T} \\
& \boldsymbol{\theta}=\left[\begin{array}{lllll}
\vartheta_{(1)} & \vartheta_{(2)} & \ldots & \vartheta_{(m-1)} & \vartheta_{(m)}
\end{array}\right]^{T} \\
& \overline{\mathbf{B}}=\left[\begin{array}{llllllll}
\bar{b}_{x(1)} & \bar{b}_{x(2)} & \ldots & \bar{b}_{x(m)} & \bar{b}_{y(1)} & \bar{b}_{y(2)} & \ldots & \bar{b}_{y(m)}
\end{array}\right]^{T} \\
& \mathbf{K}^{B B}=\left[\begin{array}{cccc}
k_{1,1}^{B B} & k_{1,2}^{B B} & \ldots & k_{1,2 m}^{B B} \\
k_{2,1}^{B B} & k_{2,2}^{B B} & \ldots & k_{2,2 m}^{B B} \\
\ldots & \ldots & \ldots & \ldots \\
k_{2 m, 1}^{B B} & k_{2 m, 2}^{B B} & \ldots & k_{2 m, 2 m}^{B B}
\end{array}\right] \\
& \mathbf{K}^{\text {dila }}=\left[\begin{array}{cccc}
k_{1,1}^{\text {dila }} & k_{1,2}^{\text {dila }} & \ldots & k_{1, m}^{\text {dila }} \\
k_{2,1}^{\text {dila }} & k_{2,2}^{\text {dila }} & \ldots & k_{2, m}^{\text {dila }} \\
\ldots & \ldots & \ldots & \ldots \\
k_{2 m, 1}^{\text {dila }} & k_{2 m, 2}^{\text {dila }} & \ldots & k_{2 m, m}^{\text {dila }}
\end{array}\right]
\end{aligned}
$$

where $\overline{\mathbf{B}}$ and $\mathbf{U}$ represent the vector of applied forces per unit area and the vector of displacements of the PD discretized model. The term $\mathbf{K}^{B B}$ represents the global stiffness matrix corresponding to the local stiffness $\mathbf{k}_{(k)(j)}^{B B}, \mathbf{K}^{\text {dila }}$ represents the global stiffness matrix corresponding to the local stiffness $\mathbf{k}_{(k)(j)}^{\text {dila }}$. Note that, the matrix $\mathbf{K}^{B B}$ given in Eq. (B3e) has a size of $(2 m \times 2 m)$. Meanwhile, $\mathbf{K}^{\text {dila }}$ given in Eq. (B3f) has a size of $(2 m \times m)$.

On the other hand, the dilatation given in Eq. (3c) can be rewritten as

$$
\vartheta_{(k)}=d \sum_{j=1}^{N_{k}} \psi_{(k)(j)} \frac{\left(u_{(j)}-u_{(k)}\right) \cos \varphi+\left(v_{(j)}-v_{(k)}\right) \sin \varphi}{\xi} V_{(j)}
$$

or

$$
\vartheta_{(k)}=\sum_{j=1}^{N_{k}} \psi_{(k)(j)} \frac{d}{\xi}\left[\begin{array}{llll}
-\cos \varphi & \cos \varphi & -\sin \varphi & \sin \varphi
\end{array}\right]\left[\begin{array}{c}
u_{(k)} \\
u_{(j)} \\
v_{(k)} \\
v_{(j)}
\end{array}\right] V_{(j)}
$$

or

$$
\vartheta_{(k)}=\sum_{j=1}^{N_{k}} \mathbf{k}_{(k)(j)}^{\vartheta}\left[\begin{array}{c}
u_{(k)} \\
u_{(j)} \\
v_{(k)} \\
v_{(j)}
\end{array}\right]
$$

with

$$
\mathbf{k}_{(k)(j)}^{\vartheta}=\psi_{(k)(j)} \frac{d}{\xi} V_{(j)}\left[\begin{array}{llll}
-\cos \varphi & \cos \varphi & -\sin \varphi & \sin \varphi
\end{array}\right]
$$


Note that Eq. (B4c) is the dilatation for material point $k$. By assuming the PD discretized model has $m$ material points, the global dilatation vector of all material points in the PD model can be calculated as

$\boldsymbol{\theta}=\left(\mathbf{K}^{\vartheta}\right) \mathbf{U}$

with

$$
\mathbf{K}^{\vartheta}=\left[\begin{array}{cccc}
k_{1,1}^{\vartheta} & k_{1,2}^{\vartheta} & \ldots & k_{1,2 m}^{\vartheta} \\
k_{2,1}^{\vartheta} & k_{2,2}^{\vartheta} & \ldots & k_{2,2 m}^{\vartheta} \\
\ldots & \ldots & \ldots & \ldots \\
k_{m, 1}^{\vartheta} & k_{m, 2}^{\vartheta} & \ldots & k_{m, 2 m}^{\vartheta}
\end{array}\right]
$$

Note that, the matrix $\mathbf{K}^{\vartheta}$ given in Eq. (B5b) has a size of $(m \times 2 m)$.

Therefore, by substituting Eq. (B5a) into Eq. (B3a), the PD equation of motion for static loading conditions can be written as

$$
\left(\left(\mathbf{K}^{\text {dila }}\right)\left(\mathbf{K}^{\vartheta}\right)+\left(\mathbf{K}^{B B}\right)\right) \mathbf{U}=\overline{\mathbf{B}}
$$

or

$$
\mathbf{K U}=\overline{\mathbf{B}}
$$

with

$$
\mathbf{K}=\left(\mathbf{K}^{\text {dila }}\right)\left(\mathbf{K}^{\vartheta}\right)+\left(\mathbf{K}^{B B}\right)
$$

where $\mathbf{K}$ represent the total stiffness matrix of the PD model.

By solving Eq. (B6b), the displacement field of the PD model is obtained. In this study, the PD simulation is implemented in MATLAB and the displacement field is obtained by using the backslash $(\backslash)$ operator. The numerical procedure for the static solution in PD is shown in Fig. 23. 


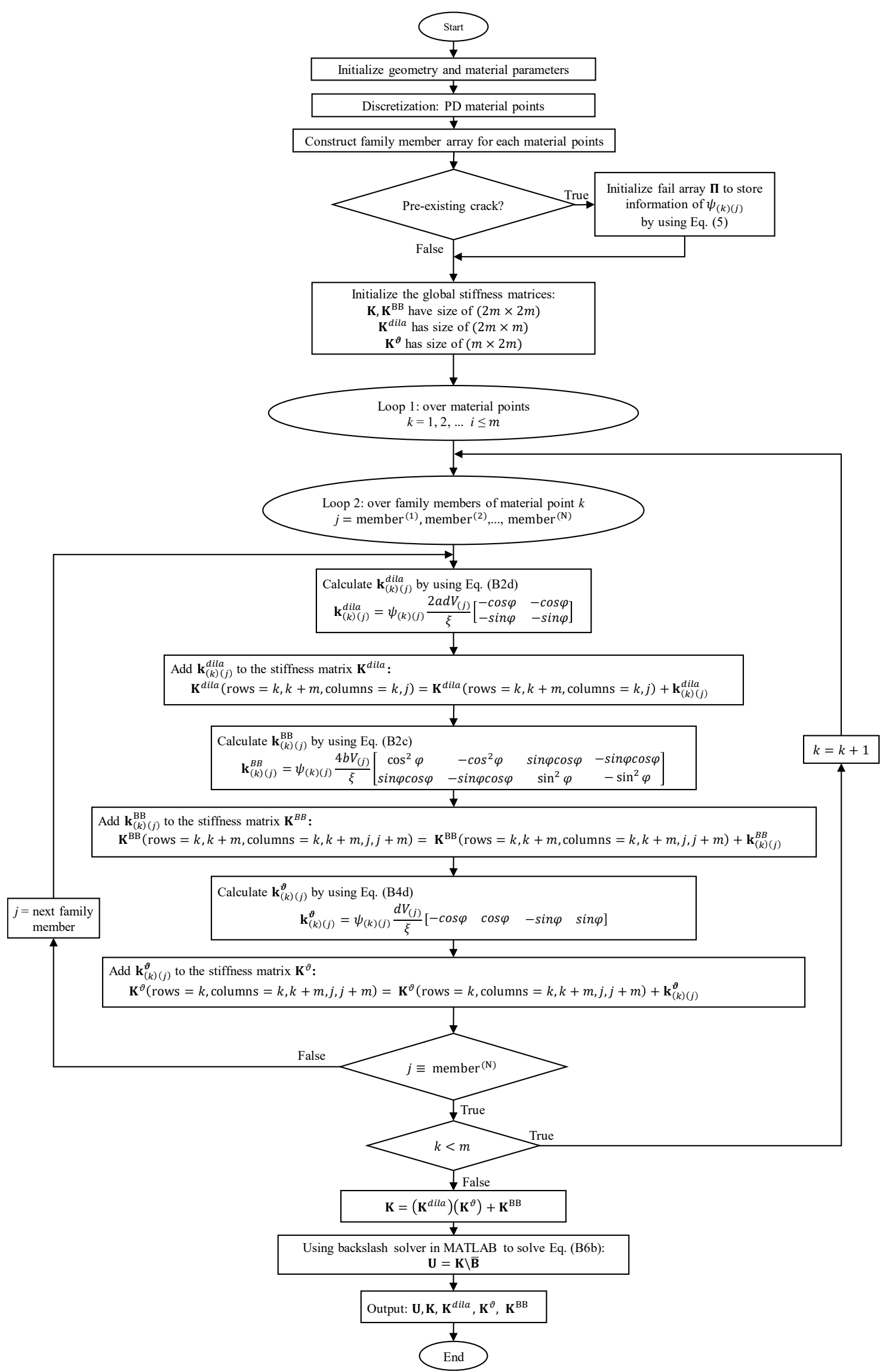

Fig. 23. Numerical procedure for the implicit solver in PD for static loading conditions 


\section{References}

[1] LR. ShipRight Design and Construction: Fatigue Design Assessment - Application and Notations (Notice 1 and Notice 2). London, UK: Lloyd's Register Group Limited; 2016. [2] LR. ShipRight Design and Construction: Fatigue Design Assessment - Level 3 Procedure Guidance on Direct Calculations (Notice 1). London, UK: Lloyd's Register Group Limited; 2016.

[3] Glenn I, Paterson R, Luznik L, Dinovitzer A, Bayley C. Fatigue resistant detail design guide for ship structures. Ship Structure Committee; 1999.

[4] DNV.GL. Fatigue assessment of ship structures. DNVGL-CG-0129: DNV GL; 2015.

[5] ClassNK. Guidelines for Direct Load Analysis and Strength Assessment. Tokyo, Japan: Development Operations Headquarters, Hull Rules Development Department, ClassNK; 2018.

[6] Veritas B. Guidelines for Fatigue Assessment of Steel Ships and Offshore Units:

Guidance Note NI 6114 DT R00 E. Neuilly sur Seine Cedex, France: Bureau Veritas, Marine \& Offshore Division; 2016.

[7] ABS. Guide for Fatigue Assessment of Offshore Structures. Houston, TX 77060 USA: American Bureau of Shipping; 2003.

[8] Thompson I. Fatigue damage variation within a class of naval ships. Ocean Engineering. 2018;165:123-30.

[9] De Gracia L, Wang H, Mao W, Osawa N, Rychlik I, Storhaug G. Comparison of two statistical wave models for fatigue and fracture analysis of ship structures. Ocean Engineering. 2019;187:106161.

[10] Yan X, Huang X, Huang Y, Cui W. Prediction of fatigue crack growth in a ship detail under wave-induced loading. Ocean Engineering. 2016;113:246-54.

[11] Li Z, Mao W, Ringsberg JW, Johnson E, Storhaug G. A comparative study of fatigue assessments of container ship structures using various direct calculation approaches. Ocean engineering. 2014;82:65-74.

[12] Kim MH, Kim SM, Kim YN, Kim SG, Lee KE, Kim GR. A comparative study for the fatigue assessment of a ship structure by use of hot spot stress and structural stress approaches. Ocean Engineering. 2009;36:1067-72.

[13] Branco R, Antunes F, Costa J. A review on 3D-FE adaptive remeshing techniques for crack growth modelling. Engineering Fracture Mechanics. 2015;141:170-95.

[14] Loghin A, Ozkan U, Kaya A, LeMonds J, McClain R, Decesare D, Akkaram S, Laflen J. 3DFAS: framework for conducting 3D crack growth simulation. Propulsion-Safety

Affordable Readiness. 2012.

[15] Pathak H, Singh A, Singh IV. Fatigue crack growth simulations of 3-D problems using XFEM. International Journal of Mechanical Sciences. 2013;76:112-31.

[16] Bhattacharya S, Singh I, Mishra B, Bui T. Fatigue crack growth simulations of interfacial cracks in bi-layered FGMs using XFEM. Computational Mechanics. 2013;52:799814.

[17] Erdogan F, Sih G. On the crack extension in plates under plane loading and transverse shear. ASME J Basic Eng. 1963; 85(4) 519-25.

[18] Hussain M, Pu S, Underwood J. Strain energy release rate for a crack under combined mode I and mode II. Fracture analysis: Proceedings of the 1973 national symposium on fracture mechanics, part II: ASTM International; 1974.

[19] Maiti SK, Smith R. Comparison of the criteria for mixed mode brittle fracture based on the preinstability stress-strain field. International Journal of Fracture. 1984;24:5-22.

[20] Eshelby JD. The force on an elastic singularity. Philosophical Transactions of the Royal Society of London Series A, Mathematical Physical Sciences. 1951;244:87-112. 
[21] Silling SA. Reformulation of elasticity theory for discontinuities and long-range forces. Journal of the Mechanics and Physics of Solids. 2000;48:175-209.

[22] Silling SA, Epton M, Weckner O, Xu J, Askari E. Peridynamic states and constitutive modeling. Journal of Elasticity. 2007;88:151-84.

[23] Silling SA, Lehoucq R. Peridynamic theory of solid mechanics. Advances in applied mechanics: Elsevier; 2010. p. 73-168.

[24] Silling SA, Askari E. A meshfree method based on the peridynamic model of solid mechanics. Computers \& structures. 2005;83:1526-35.

[25] Madenci E, Oterkus E. Peridynamic Theory and Its Applications: Springer; 2014.

[26] Mitchell JA. A non-local, ordinary-state-based viscoelasticity model for peridynamics.

Sandia National Lab Report. 2011;8064:1-28.

[27] Foster JT, Silling SA, Chen WW. Viscoplasticity using peridynamics. International journal for numerical methods in engineering. 2010;81:1242-58.

[28] Huang Y, Oterkus S, Hou H, Oterkus E, Wei Z, Zhang S. Peridynamic model for viscohyperelastic material deformation in different strain rates. Continuum Mechanics

Thermodynamics. 2019:1-35.

[29] Madenci E, Oterkus S. Ordinary state-based peridynamics for plastic deformation according to von Mises yield criteria with isotropic hardening. Journal of the Mechanics Physics of Solids. 2016;86:192-219.

[30] Madenci E, Oterkus S. Ordinary state-based peridynamics for thermoviscoelastic deformation. Engineering Fracture Mechanics. 2017;175:31-45.

[31] Nguyen CT, Oterkus S. Ordinary state-based peridynamic model for geometrically nonlinear analysis. Engineering Fracture Mechanics. 2019.

[32] Oterkus E, Madenci E. Peridynamic theory for damage initiation and growth in composite laminate. Key Engineering Materials: Trans Tech Publ; 2012. p. 355-8.

[33] Oterkus E. Peridynamic theory for modeling three-dimensional damage growth in metallic and composite structures. The University of Arizona: The University of Arizona; 2010.

[34] Hu Y, De Carvalho N, Madenci E. Peridynamic modeling of delamination growth in composite laminates. Composite Structures. 2015;132:610-20.

[35] Gao Y, Oterkus S. Fully coupled thermomechanical analysis of laminated composites by using ordinary state based peridynamic theory. Composite Structures. 2019;207:397-424.

[36] De Meo D, Zhu N, Oterkus E. Peridynamic modeling of granular fracture in polycrystalline materials. Journal of Engineering Materials Technology. 2016;138:041008.

[37] Madenci E, Oterkus S. Peridynamics for coupled field equations. Handbook of

Peridynamic Modeling. 2016:489-531.

[38] Oterkus S. Peridynamics for the solution of multiphysics problems. The University of Arizona: The University of Arizona; 2015.

[39] Askari E, Bobaru F, Lehoucq R, Parks M, Silling S, Weckner O. Peridynamics for multiscale materials modeling. Journal of Physics: Conference Series: IOP Publishing; 2008. p. 012078.

[40] Bobaru F, Ha YD. Adaptive refinement and multiscale modeling in 2D peridynamics. Journal for Multiscale Computational Engineering. 2011:635-59.

[41] Galadima Y, Oterkus E, Oterkus S. Two-dimensional implementation of the coarsening method for linear peridynamics. AIMS Materials Science. 2019;6:252-75.

[42] Nguyen CT, Oterkus S. Peridynamics formulation for beam structures to predict damage in offshore structures. Ocean Engineering. 2019;173:244-67.

[43] O'Grady J, Foster J. Peridynamic beams: a non-ordinary, state-based model.

International Journal of Solids Structures. 2014;51:3177-83. 
[44] Diyaroglu C, Oterkus E, Oterkus S. An Euler-Bernoulli beam formulation in an ordinary state-based peridynamic framework. Mathematics Mechanics of Solids. 2019;24:361-76.

[45] Diyaroglu C, Oterkus E, Oterkus S, Madenci E. Peridynamics for bending of beams and plates with transverse shear deformation. International Journal of Solids Structures.

2015;69:152-68.

[46] O'Grady J, Foster J. Peridynamic plates and flat shells: A non-ordinary, state-based model. International Journal of Solids Structures. 2014;51:4572-9.

[47] Yang Z, Oterkus E, Nguyen CT, Oterkus S. Implementation of peridynamic beam and plate formulations in finite element framework. Continuum Mechanics Thermodynamics. 2019;31:301-15.

[48] Chowdhury SR, Roy P, Roy D, Reddy J. A peridynamic theory for linear elastic shells. International Journal of Solids and Structures. 2016;84:110-32.

[49] Nguyen CT, Oterkus S. Peridynamics for the thermomechanical behavior of shell structures. Engineering Fracture Mechanics. 2019:106623.

[50] Kilic B, Madenci E. Coupling of peridynamic theory and the finite element method. Journal of mechanics of materials structures. 2010;5:707-33.

[51] Oterkus E, Madenci E, Weckner O, Silling S, Bogert P, Tessler A. Combined finite element and peridynamic analyses for predicting failure in a stiffened composite curved panel with a central slot. Composite Structures. 2012;94:839-50.

[52] Liu W, Hong J-W. A coupling approach of discretized peridynamics with finite element method. Computer methods in applied mechanics engineering. 2012;245:163-75.

[53] Bie Y, Cui X, Li Z. A coupling approach of state-based peridynamics with node-based smoothed finite element method. Computer Methods in Applied Mechanics Engineering. 2018;331:675-700.

[54] Silling SA, Askari A. Peridynamic model for fatigue cracking. SAND-18590 Albuquerque: Sandia National Laboratories. 2014.

[55] Zhang G, Le Q, Loghin A, Subramaniyan A, Bobaru F. Validation of a peridynamic model for fatigue cracking. Engineering Fracture Mechanics. 2016;162:76-94.

[56] Jung J, Seok J. Mixed-mode fatigue crack growth analysis using peridynamic approach. International Journal of Fatigue. 2017;103:591-603.

[57] Barsom J. The dependence of fatigue crack propagation on strain energy release rate and crack opening displacement. Damage Tolerance in Aircraft Structures: ASTM International; 1971.

[58] Grogan DM, Leen SB, Brádaigh CÓ. An XFEM-based methodology for fatigue delamination and permeability of composites. Composite Structures. 2014;107:205-18.

[59] Turon A, Bak B, Lindgaard E, Sarrado C, Lund E. Interface elements for fatigue-driven delaminations in advanced composite materials. Numerical modelling of failure in advanced composite materials: Elsevier; 2015. p. 73-91.

[60] Jones R, Kinloch AJ, Michopoulos JG, Brunner AJ, Phan N. Delamination growth in polymer-matrix fibre composites and the use of fracture mechanics data for material characterisation and life prediction. Composite Structures. 2017;180:316-33.

[61] Vassilopoulos A, Shahverdi M, Keller T. Mode I fatigue and fracture behavior of adhesively-bonded pultruded glass fiber-reinforced polymer (GFRP) composite joints. Fatigue and Fracture of Adhesively-Bonded Composite Joints: Elsevier; 2015. p. 149-86. [62] Raimondo A, Doesburg S, Bisagni C. Numerical study of quasi-static and fatigue delamination growth in a post-buckled composite stiffened panel. Composites Part B: Engineering. 2020;182:107589.

[63] Sarego G, Le QV, Bobaru F, Zaccariotto M, Galvanetto U. Linearized state-based peridynamics for 2-D problems. International Journal for Numerical Methods in Engineering. 2016;108:1174-97. 
[64] Diyaroglu C. Peridynamics and its applications in marine structures: University of Strathclyde; 2016.

[65] Silling SA. Linearized theory of peridynamic states. Journal of Elasticity. 2010;99:85111.

[66] Zhang H, Qiao P. A state-based peridynamic model for quantitative fracture analysis. International Journal of Fracture. 2018;211:217-35.

[67] Zhang Y, Qiao P. A new bond failure criterion for ordinary state-based peridynamic mode II fracture analysis. International Journal of Fracture. 2019;215:105-28.

[68] Foster JT, Silling SA, Chen W. An energy based failure criterion for use with peridynamic states. International Journal for Multiscale Computational Engineering. 2011;9. [69] Paris P, Erdogan F. A critical analysis of crack propagation laws. 1963.

[70] Sajith S, Murthy K, Robi P. Experimental and numerical investigation of mixed mode fatigue crack growth models in aluminum 6061-T6. International Journal of Fatigue. 2020;130:105285.

[71] MacMaster F, Chan K, Bergsma S, Kassner M. Aluminum alloy 6069 part II: fracture toughness of 6061-T6 and 6069-T6. Materials Science Engineering: A. 2000;289:54-9.

[72] McCullough R, Jordon J, Allison P, Rushing T, Garcia L. Fatigue crack nucleation and small crack growth in an extruded 6061 aluminum alloy. International Journal of Fatigue. 2019;119:52-61.

[73] Chung KH, Yang WH. Mixed mode fatigue crack growth in aluminum plates with composite patches. International journal of fatigue. 2003;25:325-33.

[74] Borrego L, Antunes F, Costa J, Ferreira J. Mixed-mode fatigue crack growth behaviour in aluminium alloy. International Journal of Fatigue. 2006;28:618-26.

[75] Standard ASTM. E647-15: Standard Test Method for Measurement of Fatigue Crack Growth Rates. West Conshohocken: ASTM International 2015. 